\title{
Cyclic Distributed Space-Time Codes for Wireless Relay Networks With No Channel Information
}

\author{
Frédérique Oggier and Babak Hassibi
}

\begin{abstract}
In this paper, we present a coding strategy for half duplex wireless relay networks, where we assume no channel knowledge at any of the transmitter, receiver, or relays. The coding scheme uses distributed space-time coding, that is, the relay nodes cooperate to encode the transmitted signal so that the receiver senses a space-time codeword. It is inspired by noncoherent differential techniques. The proposed strategy is available for any number of relays nodes. It is analyzed, and shown to yield a diversity linear in the number of relays. We also study the resistance of the scheme to relay node failures, and show that a network with $R$ relay nodes and $d$ of them down behaves, as far as diversity is concerned, as a network with $R-d$ nodes. Finally, our construction can be easily generalized to the case where the transmitter and receiver nodes have several antennas.
\end{abstract}

Index Terms-Differential modulation, distributed space-time coding, noncoherent multiple-input multiple-output (MIMO) channels.

\section{PRELIMINARIES}

W IRELESS relay networks have recently received a lot of attention. Coding strategies inspired by space-time coding techniques, where the transmit antennas cooperate to resist the fading, have been proposed, yielding cooperative diversity schemes, where the relay nodes form a virtual multipleantenna array to obtain the diversity advantage known to be achieved by multiple-input multiple-output (MIMO) systems [1], [2], [4], [5], [10], [17], [24]. These works have focused on different aspects of coding for wireless networks. In [2], the capacity of the network is computed, while a lot of work has been done on finding codes optimal with respect to the so-called diversity-multiplexing gain tradeoff [1], [5], [24].

Manuscript received March 09, 2007; revised August 24, 2009. Current version published December 23, 2009. The work of B. Hassibi was supported in part by the National Science Foundation under Grant CCF 0729203, by the Office of Naval Research under Grant N00014-08-1-0747, by the David and Lucille Packard Foundation, and by Caltech's Lee Center for Advanced Networking. The material in this paper was presented in part at the Allerton Conference on Communication, Control and Computing, Monticello, IL, September 2006 and the IEEE International Conference on Acoustics, Speech, and Signal Processing (ICASSP 2007), Honolulu, HI, 2007.

F. Oggier was with the Department of Electrical Engineering, California Institute of Technology, Pasadena, CA 91125 USA. She is now with School of Mathematical Sciences, Division of Physical and Mathematical Sciences, Nanyang Technological University, Singapore (e-mail: frederique @ systems.caltech.edu).

B. Hassibi is with Department of Electrical Engineering, California Institute of Technology, Pasadena, CA 91125 USA (e-mail: hassibi@ systems.caltech. edu).

Communicated by B. S. Rajan, Associate Editor for Coding Theory.

Color versions of Figures 2-6, 8, and 9 in this paper are available online at http://ieeexplore.ieee.org.

Digital Object Identifier 10.1109/TIT.2009.2034801

\section{A. Previous Work on Distributed Space-Time Coding}

In [10], a strategy called distributed space-time coding has been presented. The idea is to have the relay nodes apply a simple operation on their received signal, in such a way that the signal at the receiver appears as a space-time code. This involves a two-step transmission, where the transmitter first broadcasts the information to the relays, and second, the relays forward the received signal after having performed a unitary matrix multiplication on the signal. The pairwise probability of error and the diversity gain of such coding strategy has been computed. Random distributed space-time codes are used, that is, the matrices used at the relays are generated randomly.

In [12], [14], [18], distributed space-time codes following the two-step protocol of [10] which reach the diversity with good coding gain have been proposed. In [11], it was argued that in order to increase the data rate of the network, one may use multiple antennas at both the transmitter and the receiver nodes. The two-step transmission of [10] has been generalized, and the diversity and coding gain of random distributed space-time codes in the multiple-antenna nodes scenario has been computed. Algebraic distributed space-time codes for multiple-antenna nodes reaching the diversity and having a better coding gain than random codes have been presented in [19]. Recently, the setting of [10] has been generalized in [16], where the constraint of unitary matrix multiplication at the relays has been relaxed.

In [4], a different two-step protocol to implement distributed space-time code has been presented. During the first phase, the source sends a signal and the relays decode their received signal if the channel was not in outage. During the second phase, the relays which decoded cooperate to encode a space code. The receiver knows the received signals from both the phases, and decode accordingly. This gives a practical scheme, following the scheme of [17], where the code construction is based on information-theoretic arguments.

All the above strategies assume a "coherent" channel in the sense that the receiver knows all the channel paths occurring during communication. Recently, different authors started to investigate coding for a "noncoherent" channel, assuming that none of the transmitter, relay nodes, or receiver knows about the channel. A natural approach has been to recall what are the techniques used for the noncoherent MIMO channel.

\section{B. Previous Differential Coding Techniques}

Unitary differential modulation is a technique to code for noncoherent MIMO channels [7], [9]. By asking the transmitter to send at each time $t$ a codeword multiplied by what was sent at time $t-1$, differential modulation yields a decoding strategy 
that does not depend on the channel, and which is thus suitable for a noncoherent channel.

Using a similar approach for wireless networks has been investigated by several authors. In [25], differential modulation has been adapted for a decode-and-forward strategy. Differential distributed space-time coding when the nodes do not decode has been proposed independently in [13], [15], [20], in the single-antenna case. The works in [13], [15] consider a joint design of the matrices at the relays with the transmitted signal, while the work in [20] suggests a construction where the matrices at the relays are fixed first, while the transmitted signal is optimized independently.

\section{Contribution and Organization of the Paper}

In this work, we are interested in designing a coding strategy for wireless networks where we assume no channel information, that is, the transmitter and the relays are assumed not to know the channel, and the receiver decodes with no knowledge of the different paths used during communication. We furthermore consider a network where nodes are small devices with few resources, so we do not assume that they are able to decode. Instead, they just do a simple operation on the received signal for which they do not need to know the fadings. Our strategy is inspired by noncoherent MIMO unitary differential modulation. We will consider both the cases when the transmitter and receiver have one or several antennas. Our construction is valid for any number of relays, and any number of transmit/receive antennas.

We organize this paper as follows. In Section II, we start by recalling the wireless network we consider, and how distributed coding is performed. We then present a distributed coding strategy that emulates communication over a noncoherent MIMO channel. This allows us to define a differential coding strategy, described in Section III. For convenience, the idea behind differential modulation is recalled. In Section IV, we propose a mismatched decoder and show that this yields a diversity gain linear in the number of relays. We further analyze the behavior of the proposed coding strategy in case of node failures. In Section V, we discuss issues related to code constructions and we provide simulation results. Finally, we show that our construction can be generalized to the case when both the transmitter and receiver nodes have several transmit antennas.

\section{Noncoherent Distributed Space-Time Coding}

\section{A. Distributed Space-Time Codes}

Following the setting defined in [10], consider a wireless network with $R+2$ nodes which are randomly and independently distributed. Two nodes, a receiver, and a transmitter, want to communicate, while the $R$ other nodes serve as relays. Every node is equipped with a single antenna. It can transmit and receive, but not simultaneously. The channels are denoted by $f_{i}$ from the transmitter to the $i$ th relay, and by $g_{i}$ from the $i$ th relay to the receiver (see Fig. 1). Both channels are assumed independent complex Gaussian $\mathcal{C N}(0,1)$. We assume a coherence interval of length $T \geq R$ (there is no need to have more relays than coherence time, since it is shown in [10] that the diversity

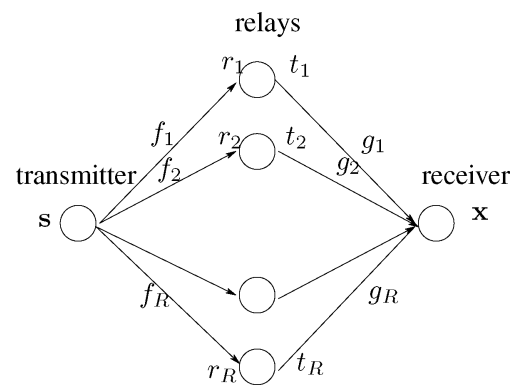

Fig. 1. The single-antenna wireless relay network model.

of the system depends on $\min \{T, R\}$ ). The total power of the system $\rho$ is equally distributed between the transmitter and the relays, so that the transmitter has an energy of $P_{1}=\rho / 2$, while each relay has $P_{2}=\rho /(2 R)$.

The transmission is done in two steps:

- Step 1: at the transmitter. Let $\boldsymbol{s}=\left(s_{1}, \ldots, s_{T}\right)^{t}$ be the signal to be sent, from the codebook $\left\{\boldsymbol{s}_{1}, \ldots, \boldsymbol{s}_{L}\right\}$ of cardinality $L$. The vector $\boldsymbol{s}$ is normalized such that $\mathrm{E}\left[\boldsymbol{s}^{\dagger} \boldsymbol{s}\right]=1$. Let $P_{1}$ be the average power available for each transmission. From time 1 to $T$, the transmitter sends the signals $\sqrt{P_{1} T} \boldsymbol{s}$ to each relay. The received signal at the $i$ th relay is given by

$$
\boldsymbol{r}_{i}=\sqrt{P_{1} T} f_{i} \boldsymbol{s}+\boldsymbol{v}_{i}, \quad i=1, \ldots, R
$$

where $\boldsymbol{v}_{i}$ is the complex Gaussian noise $\mathcal{C N}(0,1)$ at the $i$ th relay.

- Step 2: at the relays. The $i$ th relay multiplies its received signal by a unitary matrix $A_{i}$ (see Remark 1), and sends from time $T+1$ to $2 T$ the signals $\boldsymbol{t}_{i}$ to the receiver, where

$$
\boldsymbol{t}_{i}=\sqrt{\frac{P_{2}}{P_{1}+1}} A_{i} \boldsymbol{r}_{i}
$$

and the normalization factor is chosen so that $E\left[\boldsymbol{t}_{i}^{\dagger} \boldsymbol{t}_{i}\right]=$ $P_{2} T$. The signal at the receiver is given by

$$
\boldsymbol{y}=\sum_{i=1}^{R} g_{i} \boldsymbol{t}_{i}+\boldsymbol{w}
$$

where $\boldsymbol{w}$ is the complex Gaussian noise $\mathcal{C N}(0,1)$ at the receiver.

Remark 1: In order to have an equitable protocol among different users and among different times instants, the matrices $A_{i}$ are assumed unitary. This also guarantees that the noise $\boldsymbol{w}$ at the receiver remains temporally white. Note that it has been shown recently [16] that for that purpose, it is enough to require the $A_{i} \boldsymbol{v}_{i}, i=1, \ldots, R$ to be uncorrelated.

From (3), (2), and (1), the received signal is given by

$$
\boldsymbol{y}=\sqrt{\frac{P_{1} P_{2} T}{P_{1}+1}} S H+W
$$

with

$$
S=\left[A_{1} \boldsymbol{s} \cdots A_{R} \boldsymbol{s}\right], H=\left[f_{1} g_{1}, \ldots, f_{R} g_{R}\right]^{t}
$$

and

$$
W=\sqrt{\frac{P_{2}}{P_{1}+1}} \sum_{i=1}^{R} g_{i} A_{i} \boldsymbol{v}_{i}+\boldsymbol{w} .
$$


The $T \times R$ matrix $S$ works like a space-time code in a multipleantenna system. It is called a distributed space-time code since it has been generated in a distributed way by the relay nodes.

\section{B. A Noncoherent Channel}

Let us now consider the equation

$$
\boldsymbol{y}=\sqrt{\frac{P_{1} P_{2} T}{P_{1}+1}} S H+W
$$

derived above, but assume that none of the fadings are known. In a traditional noncoherent MIMO setting, it has been argued [6] that the transmitted codeword $S$ has to be unitary.

Recall that here

$$
S=\left[A_{1} s, \ldots, A_{R} s\right] .
$$

The aim is now to design the signal constellation $\left\{\boldsymbol{s}_{1}, \ldots, \boldsymbol{s}_{L}\right\} \ni$ $\boldsymbol{s}$ and the unitary matrices $A_{i}, i=1, \ldots, R$, so that the $T \times R$ matrix $S$ is unitary, i.e., $S^{\dagger} S=\boldsymbol{I}_{R}$. Let

$$
\boldsymbol{s}^{\prime}=\frac{1}{\sqrt{T}}(1, \ldots, 1)^{t}, \boldsymbol{s}_{i}=U_{i} \boldsymbol{s}^{\prime}, i=1, \ldots, L
$$

where $U_{i}$ 's are $T \times T$ unitary matrices and $\boldsymbol{s}^{\prime}$ is normalized so that $E\left[s^{\dagger} s\right]=1$.

Assume now that there exists a $T \times T$ matrix $M$ such that $M M^{\dagger}=\boldsymbol{I}_{T}$. We can then choose the matrices $A_{i}$ to be

$$
A_{i}=\operatorname{diag}\left(M_{i}\right), \quad i=1, \ldots, R
$$

where $M_{i}$ denotes a column of $M$ (recall that $T \geq R$ ). Let $U_{j}$, $j=1, \ldots, L$, be diagonal unitary matrices, thus commuting with all $A_{i}$. We have, when sending the codeword $\boldsymbol{s}_{j}$, that the corresponding distributed space-time codeword $S_{j}$ is given by

$$
S_{j}=\left[A_{1} \boldsymbol{s}_{j}, \ldots, A_{R} \boldsymbol{s}_{j}\right]=\left[U_{j} A_{1} \boldsymbol{s}^{\prime}, \ldots, U_{j} A_{R} \boldsymbol{s}^{\prime}\right]=U_{j} M / \sqrt{T}
$$

and $S_{j}^{\dagger} S_{j}=M^{\dagger} U_{j}^{\dagger} U_{j} M / T=\boldsymbol{I}_{R}$ for all transmitted signal $\boldsymbol{s}_{j}$. Let us keep in mind that the matrices $A_{i}$ have to be unitary.

Such matrices $M$ can be found in the class of Butson-Hadamard matrices (see, for example, [8]).

Definition 1: A Generalized Butson-Hadamard (GBH) matrix is a $T \times T$ matrix $M$ with coefficients in a ring such that

$$
M M^{*}=M^{*} M=T \boldsymbol{I}_{T}
$$

where $M^{*}$ is the transpose of the matrix of inverse elements of $M: m_{i j}^{*}=m_{j i}^{-1}$.

If the coefficients of $M$ are chosen to be roots of unity, then $m_{i j}^{-1}=\overline{m_{i j}}$, i.e., the inverse is the conjugate, so that

$$
M M^{\dagger}=M^{\dagger} M=T \boldsymbol{I}_{T} .
$$

Furthermore, this implies that all matrices $A_{i}$ are unitary, $i=$ $1, \ldots, R$.

Example 1: Let $\zeta_{3}=\exp (2 i \pi / 3)$ be a primitive third root of unity. Then the matrix

$$
M=\left(\begin{array}{ccc}
1 & 1 & 1 \\
1 & \zeta_{3} & \zeta_{3}^{2} \\
1 & \zeta_{3}^{2} & \zeta_{3}
\end{array}\right)
$$

is a Butson-Hadamard matrix. It is easy to check that $M M^{\dagger}=$ $3 I_{3}$. Also

$$
\begin{gathered}
\operatorname{diag}\left(M_{1}\right)=\left(\begin{array}{lll}
1 & 0 & 0 \\
0 & 1 & 0 \\
0 & 0 & 1
\end{array}\right), \operatorname{diag}\left(M_{2}\right)=\left(\begin{array}{ccc}
1 & 0 & 0 \\
0 & \zeta_{3} & 0 \\
0 & 0 & \zeta_{3}^{2}
\end{array}\right) \\
\operatorname{diag}\left(M_{3}\right)=\left(\begin{array}{ccc}
1 & 0 & 0 \\
0 & \zeta_{3}^{2} & 0 \\
0 & 0 & \zeta_{3}
\end{array}\right)
\end{gathered}
$$

are clearly unitary.

Other examples of such matrices can be found in [8]. Note that the tensor product of two GBH matrices is again a GBH matrix. This is thus a convenient way of building GBH matrices for a any dimension $T$.

Example 2: The following tensor product:

$$
M \otimes M=\left(\begin{array}{ccc}
1 & 1 & 1 \\
1 & \zeta_{3} & \zeta_{3}^{2} \\
1 & \zeta_{3}^{2} & \zeta_{3}
\end{array}\right) \otimes\left(\begin{array}{ccc}
1 & 1 & 1 \\
1 & \zeta_{3} & \zeta_{3}^{2} \\
1 & \zeta_{3}^{2} & \zeta_{3}
\end{array}\right)
$$

is a Butson-Hadamard matrix that can be used for a network with nine relay nodes.

Note that our strategy has the advantage of having independent designs for the matrices $A_{i}$ and $U_{j}$, unlike, for example, the scheme given in [15]. Thus, for a given number of relays $R$, the matrices are built once for all and given to the relays. The code design then simplifies to considering the matrices $U_{j}$. This question is addressed in Section V.

\section{A DifFERENTIAL CODING StRATEGY}

In this section, we give a strategy to implement a differential distributed coding scheme (for the sake of completeness, the standard differential scheme for MIMO channel is recalled below). It is a priori not clear how to emulate differential coding in a distributed setting. Where should the differential encoding take place? One can imagine the relays cooperating to encode differentially, similarly to the coherent case where relays encode the space-time codes, as well as having the transmitter itself collaborating with the relays. However, the construction presented in the previous section clearly suggests the approach where the differential encoder is actually at the transmitter itself. The relays cooperate not to encode differentially, but to encode a unitary space-time code.

\section{A. Differential MIMO Coding}

Consider a Rayleigh-flat fading channel with $M$ transmit antennas and $N$ receive antennas, with unknown channel information. The channel is used in blocks of $M$ channel uses, so that the transmitted signal can be represented as an $M \times M$ matrix $\boldsymbol{S}_{t}$, where $t=0,1 \ldots$ represents the block channel use. If we assume that the channel is constant over $M$ channel uses, we may write it as

$$
\boldsymbol{Y}_{t}=\sqrt{\rho} \boldsymbol{S}_{t} \boldsymbol{H}_{t}+\boldsymbol{W}_{t}, \quad t=0,1, \ldots
$$

Here $\boldsymbol{H}_{t}$, the channel matrix, and $\boldsymbol{W}_{t}$, the noise matrix, are two $M \times N$ matrices with independent complex normal coefficients, 
and $\rho$ is the expected signal-to-noise ratio (SNR) at each receiver antenna.

Differential unitary space-time modulation [7], [9] is implemented by sending at time $t$ a new codeword multiplied by the signal transmitted at time $t-1$. The transmitted signal $\boldsymbol{S}_{t}$ is thus (assuming $\boldsymbol{S}_{0}=\boldsymbol{I}$ )

$$
\boldsymbol{S}_{t}=\boldsymbol{X}_{z_{t}} \boldsymbol{S}_{t-1}, \quad t=1,2, \ldots
$$

where $z_{t} \in\{0, \ldots, L-1\}$ is the data to be transmitted, and $\mathcal{C}=\left\{\boldsymbol{X}_{0}, \ldots, \boldsymbol{X}_{L-1}\right\}$ the constellation to be designed. It can be seen from the above equation that a codebook containing unitary matrices prevents $S_{t}$ to tend either to zero or infinity. We will follow this approach, though the assumption that the matrices are unitary can be relaxed with a suitable power normalization (for example, by using scaled unitary matrices).

If we further assume the channel constant for $2 M$ consecutive uses, we get from (6) and (7) that

$$
\begin{aligned}
\boldsymbol{Y}_{t} & =\sqrt{\rho} \boldsymbol{X}_{z_{t}} \boldsymbol{S}_{t-1} \boldsymbol{H}+\boldsymbol{W}_{t} \\
& =\boldsymbol{X}_{z_{t}}\left(\boldsymbol{Y}_{t-1}-\boldsymbol{W}_{t-1}\right)+\boldsymbol{W}_{t} \\
& =\boldsymbol{X}_{z_{t}} \boldsymbol{Y}_{t-1}+\boldsymbol{W}_{t}^{\prime}
\end{aligned}
$$

where $\boldsymbol{W}_{t}^{\prime}=\boldsymbol{W}_{t}-\boldsymbol{X}_{z_{t}} \boldsymbol{W}_{t}$ is statistically independent of $\boldsymbol{X}_{z_{t}}$, since $\boldsymbol{X}_{z_{t}}$ is unitary. Since the matrix $\boldsymbol{H}$ does not appear in the last equation, this means that differential modulation allows decoding without knowledge of the channel.

Remark 2: Note that in practice, the coherence interval is usually much larger than $2 M$. In fact, what is often encountered is a continuously-fading channel. The assumption that the differential scheme exploits is that the channel is roughly constant over "any" $2 M$ channel uses.

\section{B. A Differential Encoder}

It is straightforward to adapt the two-steps transmission described in Section II-A to allow differential encoding and decoding.

Assume that the transmitter wants to send at time $t+n T$ the data $z_{t+n T}$. It is encoded into a unitary matrix $U\left(z_{t+n T}\right)$. We consider the following strategy.

1) Let $\boldsymbol{s}_{t}=U\left(z_{t}\right) \boldsymbol{s}^{\prime}$ be the signal to be transmitted, where $\boldsymbol{s}^{\prime}=(1, \ldots, 1)^{t} / \sqrt{T}$ is normalized so that $E\left[\boldsymbol{s}_{t}^{\dagger} \boldsymbol{s}_{t}\right]=1$. Let $P_{1}$ be the average energy available for each transmission. From time $t+1$ to $t+n$, the transmitter sends the signal $\sqrt{P_{1} T} \boldsymbol{s}_{t}$ to each relay. From time $t+T+1$ to $t+2 T$, the signal to be transmitted is $\boldsymbol{s}_{t+T}=U\left(z_{t+T}\right) \boldsymbol{s}_{t}$.

2) At the $i$ th relay, the received signals are (indexing the signals as a function of the time at which they have been sent)

and

$$
\boldsymbol{r}_{i}(t)=\sqrt{P_{1} T} f_{i} \boldsymbol{s}_{t}+\boldsymbol{v}_{i}(t), \quad i=1, \ldots, R
$$

$$
\begin{array}{r}
\boldsymbol{r}_{i}(t+T)=\sqrt{P_{1} T} f_{i} U\left(z_{t+T}\right) \boldsymbol{s}_{t}+\boldsymbol{v}_{i}(t+T) \\
i=1, \ldots, R
\end{array}
$$

3) The $i$ th relay multiplies its received signal by a unitary matrix $A_{i}$, where $A_{i}$ has been built using a Butson-Hadamard matrix as described in Section II-B. From time $t+T+1$ to $t+2 T$, the transmitted signal is

$$
\boldsymbol{t}_{i}(t)=\sqrt{\frac{P_{2}}{P_{1}+1}} A_{i} \boldsymbol{r}_{i}(t)
$$

and, similarly, from time $t+2 T+1$ to $t+3 T$

$$
\boldsymbol{t}_{i}(t+T)=\sqrt{\frac{P_{2}}{P_{1}+1}} A_{i} \boldsymbol{r}_{i}(t+T) .
$$

4) At time $t+2 T$, resp., $t+3 T$, the received signals are similar to (4)-(5)

$$
\begin{aligned}
\boldsymbol{y}(t) & =\sqrt{c_{\rho}} \sum_{i=1}^{R} g_{i} f_{i} A_{i} \boldsymbol{s}_{t}+W(t) \\
\boldsymbol{y}(t+T) & =\sqrt{c_{\rho}} \sum_{i=1}^{R} g_{i} f_{i} A_{i} U\left(z_{t+T}\right) \boldsymbol{s}_{t}+W(t+T)
\end{aligned}
$$

where

$$
W(t)=\sqrt{\frac{P_{2}}{P_{1}+1}} \sum_{i=1}^{R} g_{i} A_{i} \boldsymbol{v}_{i}(t)+\boldsymbol{w}(t) \text { and } c_{\rho}=\frac{P_{2} P_{1} T}{P_{1}+1} .
$$

Under the assumption that $A_{i}$ and $U\left(z_{t+T}\right)$ commute, for all $i$ and for all possible choices of $U\left(z_{t+T}\right)$, we can plug (8) into (9), which yields

$$
y(t+T)=U\left(z_{t+T}\right) y(t)+\left[W(t+T)-U\left(z_{t+T}\right) W(t)\right] .
$$

Note that the channel coefficients $f_{i}$ and $g_{i}$ do not appear in (10). Also, the assumption that $A_{i}$ and $U\left(z_{t+T}\right)$ commute is valid since both the unitary codewords $U_{j}$ and the matrices $A_{i}$ are chosen diagonal.

\section{PAIRwise Probability of ERRoR AND Nodes Failures}

\section{A. A Decoding Rule}

Emulating the point-to-point case, a natural candidate for the differential decoder is

$$
\arg \min _{U_{l}, l=1, \ldots, L}\left\|\boldsymbol{y}(t+T)-U_{l} \boldsymbol{y}(t)\right\|^{2} .
$$

Unlike in the point-to-point differential case [7], however, the above decoder is not a maximum-likelihood (ML) decoder anymore, as we shall see below.

Let us restrict to the case where $T=R$. In order to analyze this strategy, we consider two instances of the noncoherent channel

$$
y(t)=\sqrt{c_{\rho}} S(t) H+W(t)
$$

where $H$ is an $T \times T$ matrix unknown at both the transmitter and receiver, $S(t)$ is a $T \times T$ unitary matrix, and $c_{\rho}$ is a constant which depends on the $\operatorname{SNR} \rho$, that is

$$
\left(\begin{array}{c}
\boldsymbol{y}(t) \\
\boldsymbol{y}(t+T)
\end{array}\right)=\sqrt{c_{\rho}}\left(\begin{array}{c}
S(t) \\
S(t+T)
\end{array}\right) H+\left(\begin{array}{c}
W(t) \\
W(t+T)
\end{array}\right) .
$$


Since $S(t)$ and $S(t) \psi$ are indistinguishable for an arbitrary unitary $T \times T$ matrix $\psi$, we preprocess the signal so that

$$
\left(\begin{array}{c}
\boldsymbol{y}(t) \\
\boldsymbol{y}(t+T)
\end{array}\right)=\sqrt{c_{\rho}}\left(\begin{array}{c}
\boldsymbol{I}_{T} \\
U_{k}
\end{array}\right) H+\left(\begin{array}{c}
W(t) \\
W(t+T)
\end{array}\right)
$$

for $U_{k}$, a unitary matrix belonging to the codebook. To suit the network model, we have

$$
W(t)=\sqrt{\frac{P_{2}}{P_{1}+1}} \sum_{i=1}^{T} A_{i} g_{i} \boldsymbol{v}_{i}(t)+\boldsymbol{w}(t)
$$

and $H=D_{g} f$, where $D_{g}=\operatorname{diag}\left(g_{1}, \ldots, g_{T}\right)$ and $\boldsymbol{f}=\left(f_{1}, \ldots, f_{T}\right)^{t}$. Furthermore, we have $c_{\rho}=\frac{P_{2} P_{1} T}{P_{1}+1}$, and we denote $c_{\rho}^{\prime}=\frac{P_{2}}{P_{1}+1}$. Recall that $P_{1}=\rho / 2, P_{2}=\rho /(2 R)$, and $\rho$ is the total power of the system. Because of the two-step transmission, both the noise $W(t)$ and the channel matrix $H$ contain products of Gaussian random variables, which makes the decoder (11) a mismatched decoder instead of an ML decoder (where both the noise and the channel matrix are Gaussian). We will give an exact analysis of this mismatched decoder below, and show that it already gives the diversity.

\section{B. Pairwise Probability of Error}

Let us now compute the pairwise error probability $P\left(U_{k} \rightarrow\right.$ $U_{l}$ ) of decoding with the mismatched decoder (11). To compute this probability exactly, we note that

$$
P\left(U_{k} \rightarrow U_{l}\right)=E_{\boldsymbol{g}} P\left(U_{k} \rightarrow U_{l} \mid \boldsymbol{g}\right) .
$$

Let $\boldsymbol{I}_{n}$ denote the identity matrix of size $n$. Knowing $\boldsymbol{g}=$ $\left(g_{1}, \ldots, g_{T}\right)^{t}$, we have

$$
\begin{aligned}
& E\left[W(t) W(t)^{\dagger}\right] \\
& =E\left[W(t+T) W(t+T)^{\dagger}\right] \\
& =c_{\rho}^{\prime} E\left[\sum_{i, j=1}^{T} A_{i} g_{i} \boldsymbol{v}_{i}(t)\left(A_{j} g_{j} \boldsymbol{v}_{j}(t)\right)^{\dagger}\right]+E\left[W(t) W(t)^{\dagger}\right] \\
& =c_{\rho}^{\prime} \sum_{i=1}^{T} A_{i} A_{i}^{\dagger}\left|g_{i}\right|^{2} \boldsymbol{I}_{T}+\boldsymbol{I}_{T} \\
& =\left(c_{\rho}^{\prime}\|g\|^{2}+1\right) \boldsymbol{I}_{T} .
\end{aligned}
$$

Letting $\boldsymbol{y}=\left[\begin{array}{lll}\boldsymbol{y}(t) & \boldsymbol{y}(t+T)\end{array}\right]^{t}$, we have

$$
\begin{aligned}
\Sigma & :=E\left[\boldsymbol{y} \boldsymbol{y}^{\dagger}\right] \\
& =\left(\begin{array}{cc}
c_{\rho} D_{|g|}+\left(c_{\rho}^{\prime}\|\boldsymbol{g}\|^{2}+1\right) \boldsymbol{I}_{T} & c_{\rho} D_{|g|} U_{k}^{\dagger} \\
c_{\rho} D_{|g|} U_{k} & c_{\rho} D_{|g|}+\left(c_{\rho}^{\prime}\|\boldsymbol{g}\|^{2}+1\right) \boldsymbol{I}_{T}
\end{array}\right)
\end{aligned}
$$

where $D_{|g|}=\operatorname{diag}\left(\left|g_{1}\right|^{2}, \ldots,\left|g_{T}\right|^{2}\right)$.

The pairwise probability of error is given by

$$
\begin{aligned}
& P\left(U_{k} \rightarrow U_{l}\right) \\
& =P\left(\left\|\boldsymbol{y}(t+T)-U_{k} \boldsymbol{y}(t)\right\|^{2} \geq\left\|\boldsymbol{y}(t+T)-U_{l} \boldsymbol{y}\right\|^{2} \mid U_{k} \text { sent }\right) \\
& =P\left(\left\|\left[-U_{k} \boldsymbol{I}_{T}\right] \boldsymbol{y}\right\|^{2} \geq\left\|\left[-U_{l} \boldsymbol{I}_{T}\right] \boldsymbol{y}\right\|^{2} \mid U_{k} \text { is sent }\right) \\
& =P\left(\boldsymbol{y}^{\dagger} U \boldsymbol{y} \geq 0 \mid U_{k} \text { is sent }\right)
\end{aligned}
$$

with $k \neq l$ and

$$
\boldsymbol{U}=\left(\begin{array}{cc}
0 & U_{l}^{\dagger}-U_{k}^{\dagger} \\
U_{l}-U_{k} & 0
\end{array}\right)
$$

We start by computing $P\left(\boldsymbol{y}^{\dagger} \boldsymbol{U} \boldsymbol{y} \geq 0\right)$ knowing $\boldsymbol{g}$.
Proposition 1: We have that at high SNR

$$
\begin{aligned}
& P\left(\boldsymbol{y}^{\dagger} \boldsymbol{U} \boldsymbol{y} \geq 0 \mid \boldsymbol{g}\right) \\
& \quad \leq \frac{1}{2} \operatorname{det}\left(\boldsymbol{I}_{T}+\frac{c_{\rho}}{8\left(\frac{1}{T}\|g\|^{2}+1\right)} D_{|g|}\left(U_{k}-U_{l}\right)\left(U_{k}-U_{l}\right)^{\dagger}\right)^{-1} .
\end{aligned}
$$

Proof: We have

$$
\begin{aligned}
P\left(\boldsymbol{y}^{\dagger} \boldsymbol{U} \boldsymbol{y} \geq 0 \mid \boldsymbol{g}\right) & =E\left[u\left(\boldsymbol{y}^{\dagger} \boldsymbol{U} \boldsymbol{y}\right)\right] \\
& =E\left[\frac{1}{2 \pi} \int_{-\infty}^{\infty} \frac{e^{i \omega \boldsymbol{y}^{\dagger} \boldsymbol{U} \boldsymbol{y}}}{i \omega} d \omega\right]
\end{aligned}
$$

where $u$ is the step function $(u(x)=1$ if $x>0, u(x)=$ 0 else), and the second equality is the Fourier transform of $u$. Computing the expectation yields

$$
\begin{aligned}
P\left(\boldsymbol{y}^{\dagger} \boldsymbol{U} \boldsymbol{y} \geq 0 \mid \boldsymbol{g}\right) & =\frac{1}{2 \pi} \iint_{-\infty}^{\infty} \frac{e^{i \omega \boldsymbol{y}^{\dagger} \boldsymbol{U} \boldsymbol{y}}}{i \omega} \frac{e^{-\boldsymbol{y}^{\dagger} \Sigma^{-1} \boldsymbol{y}}}{(2 \pi)^{2 T} \operatorname{det}(\Sigma)} d \omega d \boldsymbol{y} \\
& =\frac{1}{(2 \pi)^{2 T+1} i} \int_{-\infty}^{\infty} \int \frac{e^{-\boldsymbol{y}^{\dagger}\left(-i \omega \boldsymbol{U}+\Sigma^{-1}\right) \boldsymbol{y}}}{\omega \operatorname{det}(\Sigma)} d \boldsymbol{y} d \omega .
\end{aligned}
$$

Since the exponent of the exponential is of the form $i \boldsymbol{y}^{\dagger} \omega \boldsymbol{U} \boldsymbol{y}-$ $\boldsymbol{y}^{\dagger} \Sigma^{-1} \boldsymbol{y}$, with real part $-\boldsymbol{y}^{\dagger} \Sigma^{-1} \boldsymbol{y}$ which is negative, and imaginary part given by $i \boldsymbol{y}^{\dagger} \omega \boldsymbol{U} \boldsymbol{y}$ (recall that $\boldsymbol{U}$ is Hermitian), then this integral converges and we have

$$
P\left(\boldsymbol{y}^{\dagger} \boldsymbol{U} \boldsymbol{y} \geq 0 \mid \boldsymbol{g}\right)=\frac{1}{2 \pi i} \int_{-\infty}^{\infty} \frac{1}{\omega \operatorname{det}(\boldsymbol{I}-i \omega \boldsymbol{U} \Sigma)} d \omega .
$$

We have that the above determinant is given by

$$
\begin{aligned}
\prod_{k=1}^{T}\left(1+i \omega c_{\rho}\left|g_{k}\right|^{2} \mid u_{i k}-\right. & \left.u_{j k}\right|^{2} \\
& \left.+\omega^{2}\left|u_{i k}-u_{j k}\right|^{2}\left(2 a c_{\rho}\left|g_{k}\right|^{2}+a^{2}\right)\right)
\end{aligned}
$$

where $a=c_{\rho}^{\prime}\|\boldsymbol{g}\|^{2}+1$. Since our goal is a diversity computation, we are interested in an very high SNR regime. Note that when $\rho$ is big, $c_{\rho}^{\prime}=\frac{P_{2}}{P_{1}+1}=\frac{\rho}{T(\rho+2)} \rightarrow 1 / T$. Thus, $a \rightarrow\|g\|^{2} / T+1$ and the term in $a^{2}$ does not depend on $\rho$, so that we have

$\operatorname{det}(\boldsymbol{I}-i \omega \boldsymbol{U} \Sigma) \approx$

$\prod_{k=1}^{T} c_{\rho}\left|g_{k}\right|^{2}\left|u_{i k}-u_{j k}\right|^{2}\left(\frac{1}{c_{\rho}\left|g_{k}\right|^{2}\left|u_{i k}-u_{j k}\right|^{2}}+i \omega+\omega^{2} 2 a\right)$.

By completing the squares, we get that $P\left(\boldsymbol{y}^{\dagger} \boldsymbol{U} \boldsymbol{y} \geq 0 \mid \boldsymbol{g}\right)$ is given by

$$
\begin{aligned}
\frac{1}{2 \pi i} \int_{-\infty}^{\infty} \frac{1}{\omega}\left(\prod_{k=1}^{T} c_{\rho}\left|g_{k}\right|^{2} \mid u_{i k}-\right. & \left.u_{j k}\right|^{2} 2 a \\
& {\left.\left[\left(\omega+\frac{i}{4 a}\right)^{2}+c_{k}^{2}\right]\right)^{-1} d \omega }
\end{aligned}
$$

where

$$
c_{k}:=\sqrt{\frac{1}{16 a^{2}}+\frac{1}{c_{\rho}\left|g_{k}\right|^{2}\left|u_{i k}-u_{j k}\right|^{2}}} .
$$


Note that the above integral has poles in $\omega=-i\left(1 / 4 a \pm c_{k}\right)$. Thus, as long as $-i\left(1 / 4 a-c_{k}\right)<\operatorname{Im}(\omega)<-i\left(1 / 4 a+c_{k}\right)$, the above integral is well defined. We thus choose the following contour of integration, within the convergence region:

$$
\begin{aligned}
\frac{1}{2 \pi i} \int_{-\infty-\frac{i}{4 a}}^{\infty-\frac{i}{4 a}} \frac{1}{\omega}\left(\prod_{k=1}^{T} c_{\rho}\left|g_{k}\right|^{2}\left|u_{i k}-u_{j k}\right|^{2} 2 a\right. \\
\\
{\left.\left[\left(\omega+\frac{i}{4 a}\right)^{2}+c_{k}^{2}\right]\right)^{-1} d \omega }
\end{aligned}
$$

and with a change of variable, we get

$$
\begin{aligned}
\frac{1}{2 \pi i} \int_{-\infty}^{\infty} \frac{1}{\omega-\frac{i}{4 a}}\left(\prod_{k=1}^{T} c_{\rho}\left|g_{k}\right|^{2}\left|u_{i k}-u_{j k}\right|^{2} 2 a .\right. \\
{\left.\left[\omega^{2}+\frac{1}{16 a^{2}}+\frac{1}{c_{\rho}\left|g_{k}\right|^{2}\left|u_{i k}-u_{j k}\right|^{2}}\right]\right)^{-1} d \omega . }
\end{aligned}
$$

Following [6], we obtain a bound on the probability of error that we know real by taking the real part of the above expression

$$
\begin{aligned}
& P\left(\boldsymbol{y}^{\dagger} U \boldsymbol{y} \geq 0 \mid \boldsymbol{g}\right) \\
& \lesssim \frac{1}{2 \pi} \int_{-\infty}^{\infty} \frac{4 a}{1+16 a^{2} \omega^{2}}\left(\prod_{k=1}^{T} c_{\rho}\left|g_{k}\right|^{2}\left|u_{i k}-u_{j k}\right|^{2} 2 a .\right. \\
& \left.\leq \frac{1}{2 \pi} \int_{-\infty}^{\infty} \frac{4 a d \omega}{1+16 a^{2} \omega^{2}}\left(\prod_{k=1}^{T}+\frac{1}{16 a^{2}}\right]+1\right)^{-1} d \omega \\
& =\frac{1}{2}\left(\prod_{k=1}^{T} c_{\rho}\left|g_{k}\right|^{2}\left|u_{i k}-u_{i k}-u_{j k}\right|^{2} \frac{1}{8 a} \frac{1}{8 a}+1\right)^{-1} \\
& =\frac{1}{2} \operatorname{det}\left(\boldsymbol{I}_{T}+\frac{c_{\rho}}{8\left(\frac{1}{T}\|g\|^{2}+1\right)} D_{|g|}\left(U_{k}-U_{l}\right)\left(U_{k}-U_{l}\right)^{\dagger}\right)^{-1} .
\end{aligned}
$$

Thus

$$
\begin{aligned}
& P\left(U_{k} \rightarrow U_{l}\right) \\
& \quad=E_{\boldsymbol{g}} P\left(\boldsymbol{y}^{\dagger} \boldsymbol{U} \boldsymbol{y} \geq 0 \mid \boldsymbol{g}\right) \\
& \leq E_{\boldsymbol{g}} \operatorname{det}\left(\boldsymbol{I}_{T}+\frac{c_{\rho}}{8\left(\frac{1}{T}\|g\|^{2}+1\right)} D_{|g|}\left(U_{k}-U_{l}\right)\left(U_{k}-U_{l}\right)^{\dagger}\right)^{-1} .
\end{aligned}
$$

This bound on the pairwise probability of error is similar to the Chernoff bound obtained in [10, Theorem 1], where it has been proven, by computing the above expectation on $\boldsymbol{g}=\left(g_{1}, \ldots, g_{T}\right)$, that the diversity gain is given by

$$
\operatorname{rank}\left(\left(U_{k}-U_{l}\right)\left(U_{k}-U_{l}\right)^{\dagger}\right)\left(1-\frac{\log \log P}{\log P}\right) .
$$

Thus, when $\left(U_{k}-U_{l}\right)\left(U_{k}-U_{l}\right)^{\dagger}$ is full rank (that is, the code is fully diverse), we get a diversity of

$$
R\left(1-\frac{\log \log P}{\log P}\right) \text {. }
$$

\section{Analysis of Node Failures}

In our network setting, relay nodes are small devices with a limited amount of power. It is thus very likely that some of them may run out of battery, or may encounter a technical problem that will prevent them from communicating at a given point of the transmission. In this subsection, we are interested in understanding how a coding strategy designed for $R$ relay nodes will behave if $d$ of them are down.

Let us consider one step of the differential scheme, thus involving two transmissions, say $\boldsymbol{y}(t)$ and $\boldsymbol{y}(t+T)$. Let us assume that $\boldsymbol{y}(t)$ is transmitted correctly, but one or several node failures happen while transmitting $\boldsymbol{y}(t+T)$. Denote by $\mathcal{D}$ the set of indices of relay nodes which are down during the transmission of $\boldsymbol{y}(t+T)$. In (9) for $\boldsymbol{y}(t+T)$ we thus have

$$
\sum_{i=1}^{R} g_{i} f_{i} A_{i} U\left(z_{t+T}\right) \boldsymbol{s}_{t}=\sum_{i \notin \mathcal{D}} g_{i} f_{i} A_{i} U\left(z_{t+T}\right) \boldsymbol{s}_{t} .
$$

In (8) for $\boldsymbol{y}(t)$, we can similarly write

$$
\sum_{i=1}^{R} g_{i} f_{i} A_{i} \boldsymbol{s}_{t}=\sum_{i \notin \mathcal{D}} g_{i} f_{i} A_{i} \boldsymbol{s}_{t}+\sum_{i \in \mathcal{D}} g_{i} f_{i} A_{i} \boldsymbol{s}_{t}
$$

so that when we plug (8) inside (9), the term $\sum_{i \in \mathcal{D}} g_{i} f_{i} A_{i} \boldsymbol{s}_{t}$ adds to the noise, and the codeword $U_{z_{t+T}}$ will not be recovered.

However, during the next step, things are different. Consider now the step involving $\boldsymbol{y}(t+T)$ and $\boldsymbol{y}(t+2 T)$. Now, for both signals, the same set of relay nodes will be down. Thus, communication will take place over a network where actually $R-|\mathcal{D}|$ are transmitting. The expression for $\boldsymbol{y}(t+T)$ and $\boldsymbol{y}(t+2 T)$ are similar to those obtained in (8) and (9), except that the sums are over $i \in R \backslash \mathcal{D}$ instead of $i=1, \ldots, R$. We thus expect the diversity of such systems to be

$$
(R-d)\left(1-\frac{\log \log P}{\log P}\right)
$$

where $d=|\mathcal{D}|$. This is a positive result, since it says that the strategy is resistant to node failures.

\section{Code Constructions and Simulation Results}

In this section, we first consider code constructions, and show that though any random diagonal unitary matrix could be used to generate a codebook, codes designed to optimize the diversity product reach a much better coding gain. We then provide simulation results, also including the node failures scenario. We finally discuss the decoding issue.

\section{A. Cyclic Codes}

Since the matrices $A_{i}$ used at the relays are fixed for a given number of relays $R$, designing codes consists of constructing the diagonal unitary matrices $U(z)$ in which the data $z$ to be sent will be encoded. Regarding the pairwise error probability (PEP) computation (13), the matrices $U(z)$ have to satisfy the full diversity criterion

$$
\operatorname{det}\left(U\left(z_{t}\right)-U\left(z_{t^{\prime}}\right)\right) \neq 0, \quad t \neq t^{\prime}
$$




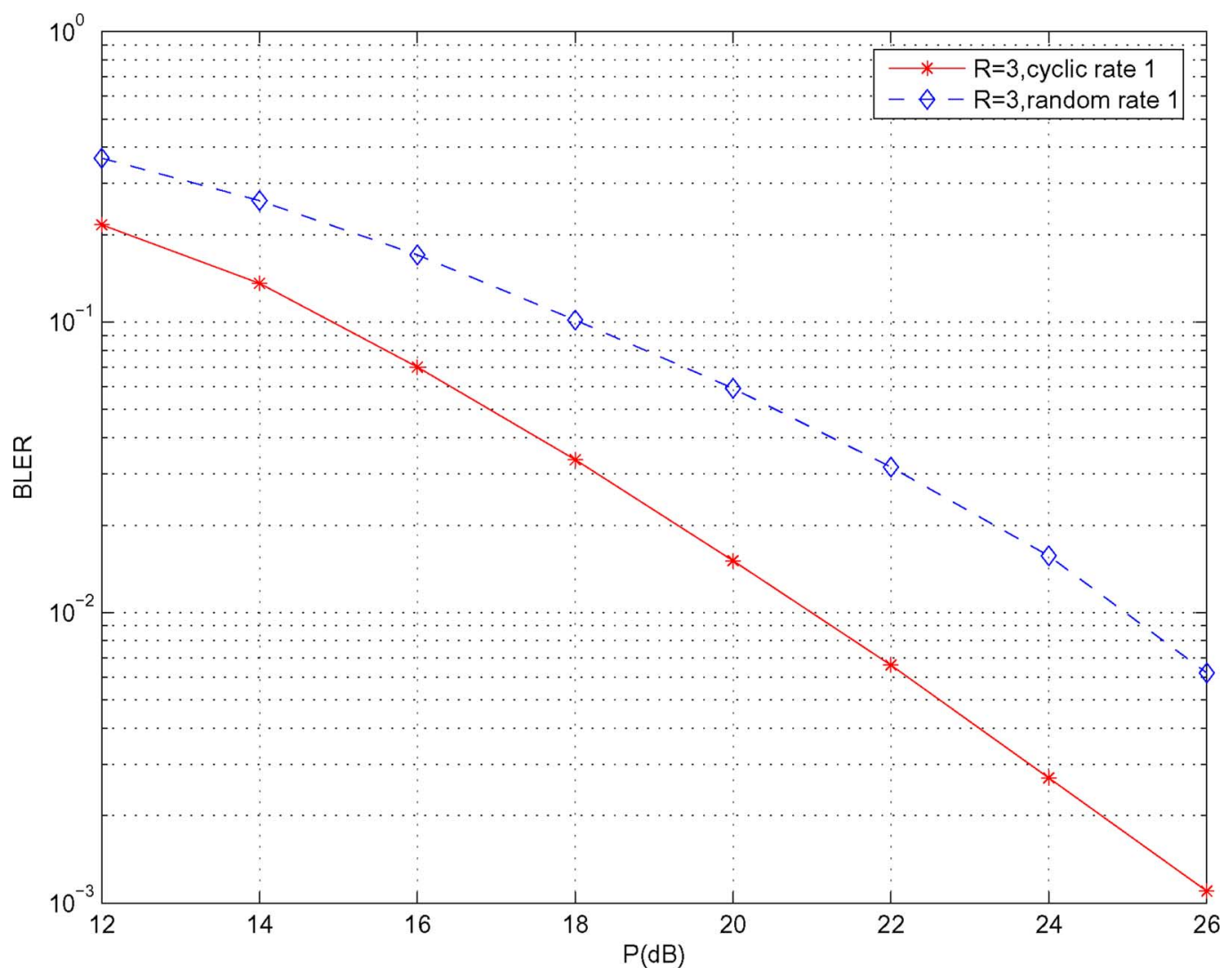

Fig. 2. A random rate 1 code versus a cyclic rate 1 code.

A priori, any diagonal unitary matrix could be use to build the code, since random unitary diagonal matrices yield full diversity with probability 1 . This makes our scheme attractive since it is thus available easily for any number of relay nodes $R$.

In order now to reach a good coding gain, one need, similarly to the point-to-point case, to maximize the diversity product, given by

$$
\zeta(U)=\min _{t \neq t^{\prime}} \frac{1}{2}\left|\operatorname{det}\left(U\left(z_{t}\right)-U\left(z_{t^{\prime}}\right)\right)\right|^{1 / R} .
$$

Diagonal unitary codes achieving good diversity product have already been studied in [7] for the MIMO case. They are called cyclic codes. Denote by $\zeta_{L}=\exp (2 i \pi / L)$ a primitive $L$ th root of unity. A cyclic code is parameterized as follows:

$$
\left(\begin{array}{ccc}
\zeta_{L}^{u_{1} l} & & 0 \\
0 & \ddots & 0 \\
0 & & \zeta_{L}^{u_{R} l}
\end{array}\right), \quad l=0, \ldots, L-1
$$

where $L$ and $u=\left(u_{1}, \ldots, u_{R}\right)$ have to be designed. Note that $R$ denotes the number of antennas in the point-to-point case, or the number of relays in the distributed case. Let us give an example with three relay nodes. Let $\zeta_{63}=\exp (2 i \pi / 63)$. The codebook is given by

$$
\left\{D^{i}, D=\left(\begin{array}{ccc}
\zeta_{63} & 0 & 0 \\
0 & \zeta_{63}^{17} & 0 \\
0 & 0 & \zeta_{63}^{26}
\end{array}\right)\right\}
$$

for $i=1, \ldots, 63$. Thus, $L=63$ and $u=(1,17,26)$.
TABLE I

Some CyClic Codes With Good Diversity Product

\begin{tabular}{|c|c|c|c|c|}
\hline$R$ & $L$ & rate & $\zeta(U)$ & $u$ \\
\hline 3 & 8 & 1 & 0.5134 & $(1,1,3)$ \\
3 & 63 & 1.99 & 0.3301 & $(1,17,26)$ \\
6 & 64 & 1 & 0.3792 & $(1,7,15,23,25,31)$ \\
6 & 4096 & 2 & 0.1428 & $(1,599,623,1445,1527,1715)$ \\
9 & 57 & 0.65 & 0.361 & $(1,4,16,7,28,55,49,25,43)$ \\
\hline
\end{tabular}

Table I summarizes some good cyclic codes. The codes for three and six relays have been found in [7], where cyclic codes with maximized coding gain are available until dimension 6 only. ${ }^{1}$ The code for nine relays is the diagonal component of a fixed-point free group based code [23].

In Fig. 2, we compare a random code with a cyclic code. We plot the block error rate (BLER) as a function of the power of the system in decibels. We consider the cyclic code $u=(1,1,3)$ with rate 1 , with a random code of rate 1 . The random code consists of generating one random unitary diagonal matrix and take its powers from 1 to 8 . The relays $R_{i}, i=1,2,3$, use, respectively, the matrices

$$
A_{1}=\boldsymbol{I}_{3}, A_{2}=\left(\begin{array}{ccc}
1 & 0 & 0 \\
0 & \zeta_{3} & 0 \\
0 & 0 & \zeta_{3}^{2}
\end{array}\right), A_{3}=\left(\begin{array}{ccc}
1 & 0 & 0 \\
0 & \zeta_{3}^{2} & 0 \\
0 & 0 & \zeta_{3}
\end{array}\right) .
$$

Both codes are fully diverse, but clearly, the cyclic code with high diversity product reaches a much better coding gain.

${ }^{1}$ After the submission of this paper, some cyclic codes in higher dimensions have been proposed in [22]. 


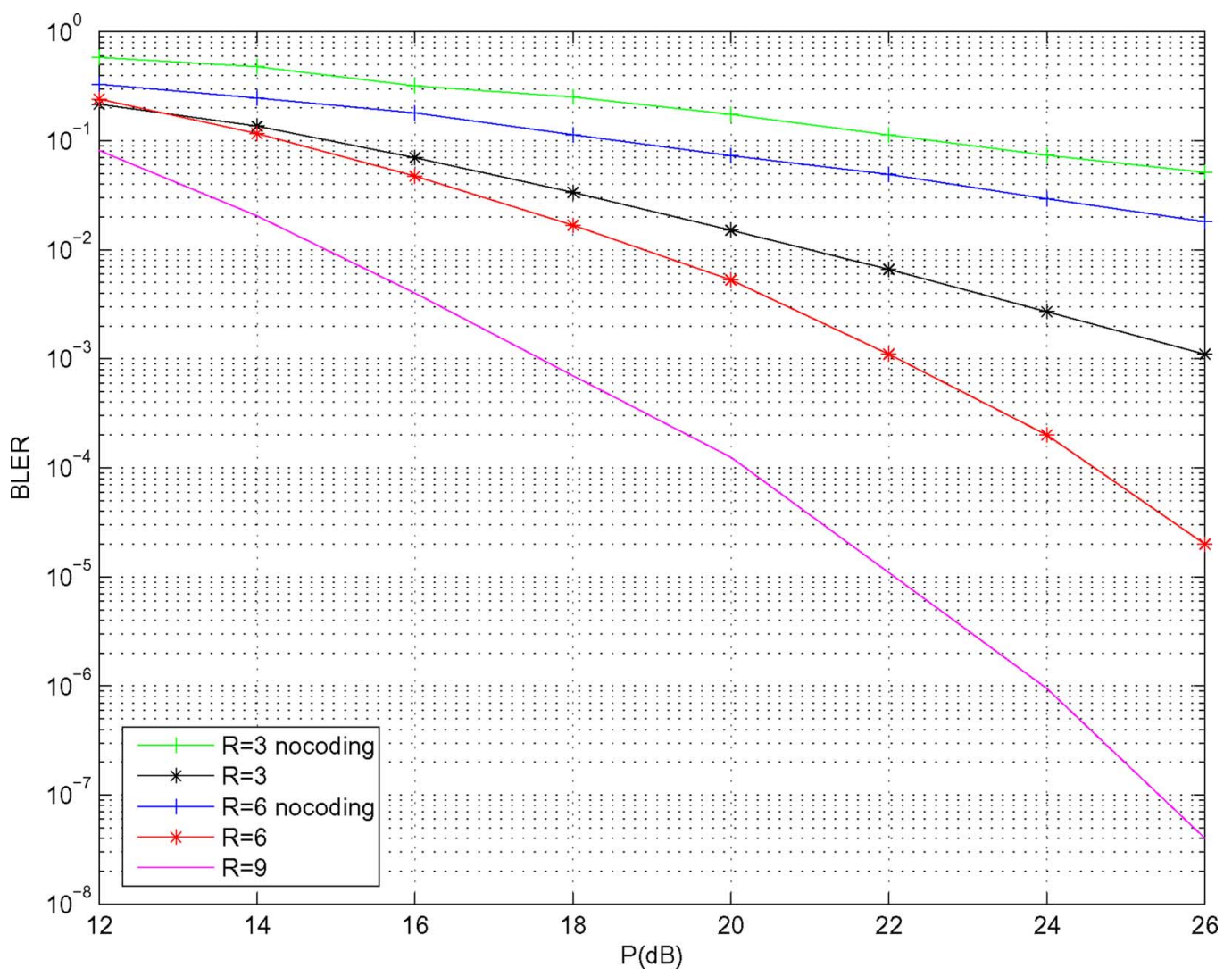

Fig. 3. Rate 1 cyclic codes: with and without coding at the relays.

On the one hand, there is thus a real coding gain that could be achieved by choosing carefully the diagonal matrices. On the other hand, since random unitary diagonal matrices yield full diversity, the differential coding scheme we propose is available for any number of relay nodes.

\section{B. Illustration of the Diversity}

Simulation results in Figs. 3, 4, and in Fig. 5 show the BLER as a function of $P$, the power of the system in decibels. To start with, Fig. 3 first shows what we already know: there is really a need for coding at the relays, without which we cannot get diversity. We see clearly that without coding at the relays, having three or six relays in the system does not change the curve and thus gives no diversity.

Recall that the analysis of the PEP in (13) predicts a diversity which is linear in the number of relays. Fig. 3 further shows how the diversity is increasing, by going from three to six, and then to nine relays. The codes used are the two cyclic rate 1 codes given in Table I for, respectively, three and six relays, and the cyclic code for nine relays with rate 0.65 .

Fig. 4 shows simulation results for rate 2 cyclic codes, with, respectively, three and six relay nodes. Clearly, increasing the rate decreases the performance, and in particular, the slope given by the diversity starts at high power. Note that this crossing of the two curves was already observed in the point-to-point case [7], but at a lower SNR.

Finally, Fig. 5 provides a comparison with other proposed codes, respectively, in [13], [15]. The code proposed in [15] has been optimized and is performing better. However, the optimization method is particular to this code. By contrast, the framework we propose is general, and if one is interested in optimizing a given construction, this can be done independently of the coding strategy at the relays.

\section{Resistance to Node Failures}

We finally give simulation results to illustrate the behavior in case of node failures. The analysis done in Section IV shows that as far as diversity is concerned, a network of $R$ relay nodes having $d$ relays not communicating behaves as a network with $R-d$ nodes. This behavior can be observed in Fig. 6. On top, the performance of a network with six relay nodes is shown. Simulations are then done, assuming that one, then two, and finally three relay nodes are down. We see how the curves change linearly with the number of relay nodes.

Fig. 6 at the bottom compares a network with six relay nodes, but only three of them communicating, with a network having only three nodes. We observe the same diversity, which was predicted by the analysis in (14). However, there is a clear loss in coding gain, which is expected.

The simulation results of this section thus confirm good behavior of the proposed strategy when facing node failures.

\section{Decoding Issues}

We finally briefly discuss decoding issues. Simulations shown in this paper have been done via exhaustive search. Indeed, for such rates, there is no real complexity problem in doing an exhaustive search. However, when increasing the 


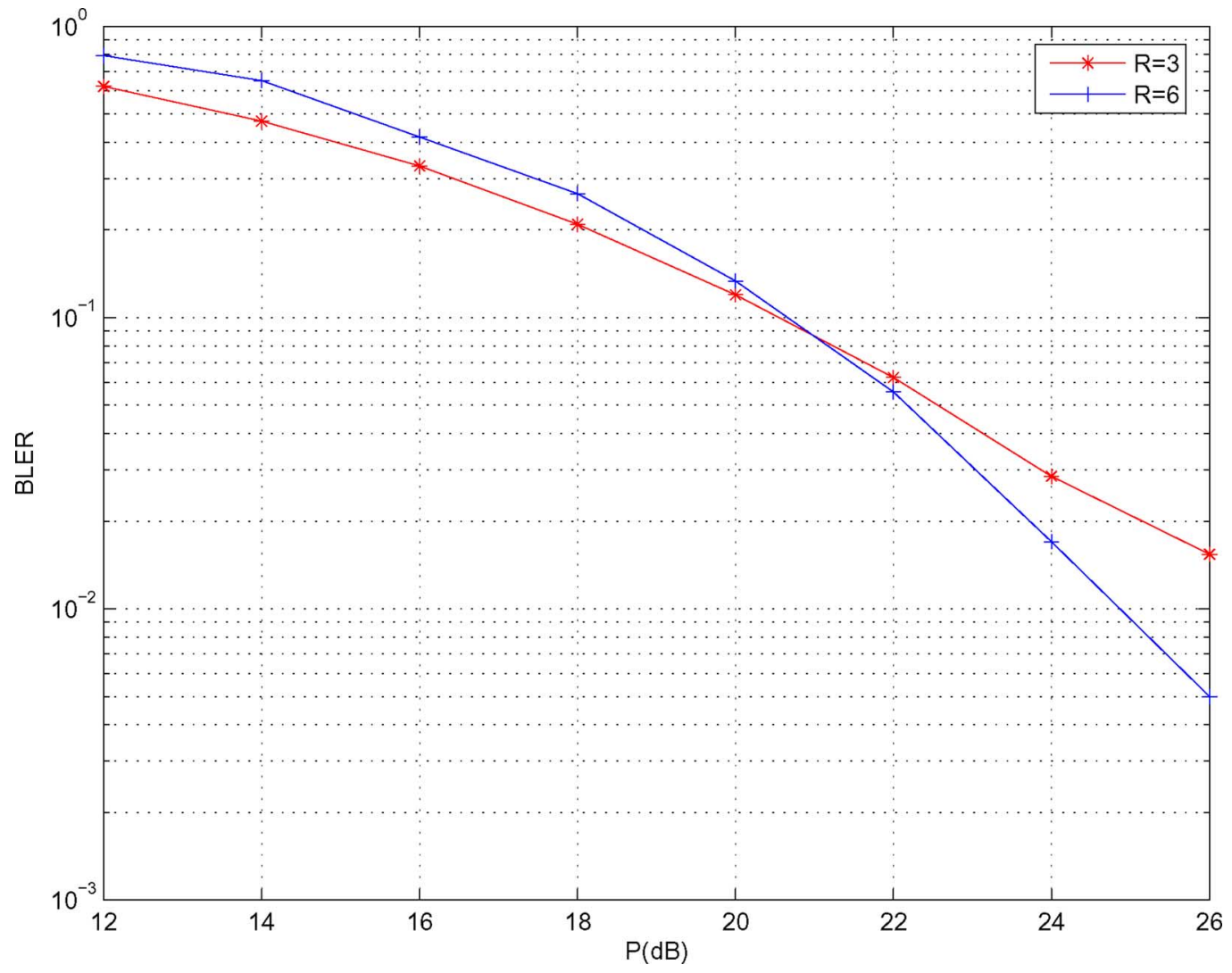

Fig. 4. Rate 2 cyclic codes.

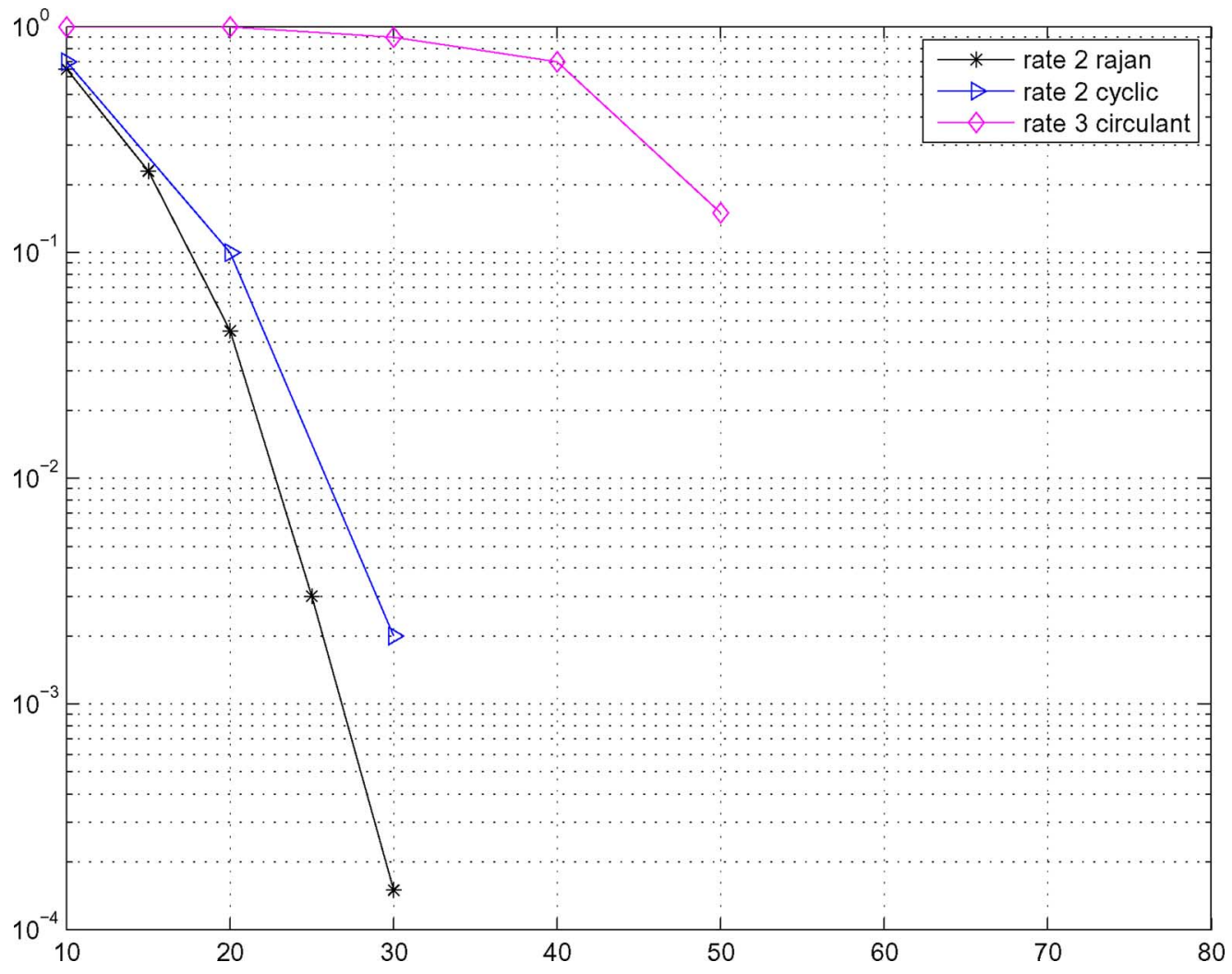

Fig. 5. Comparison with other codes. 

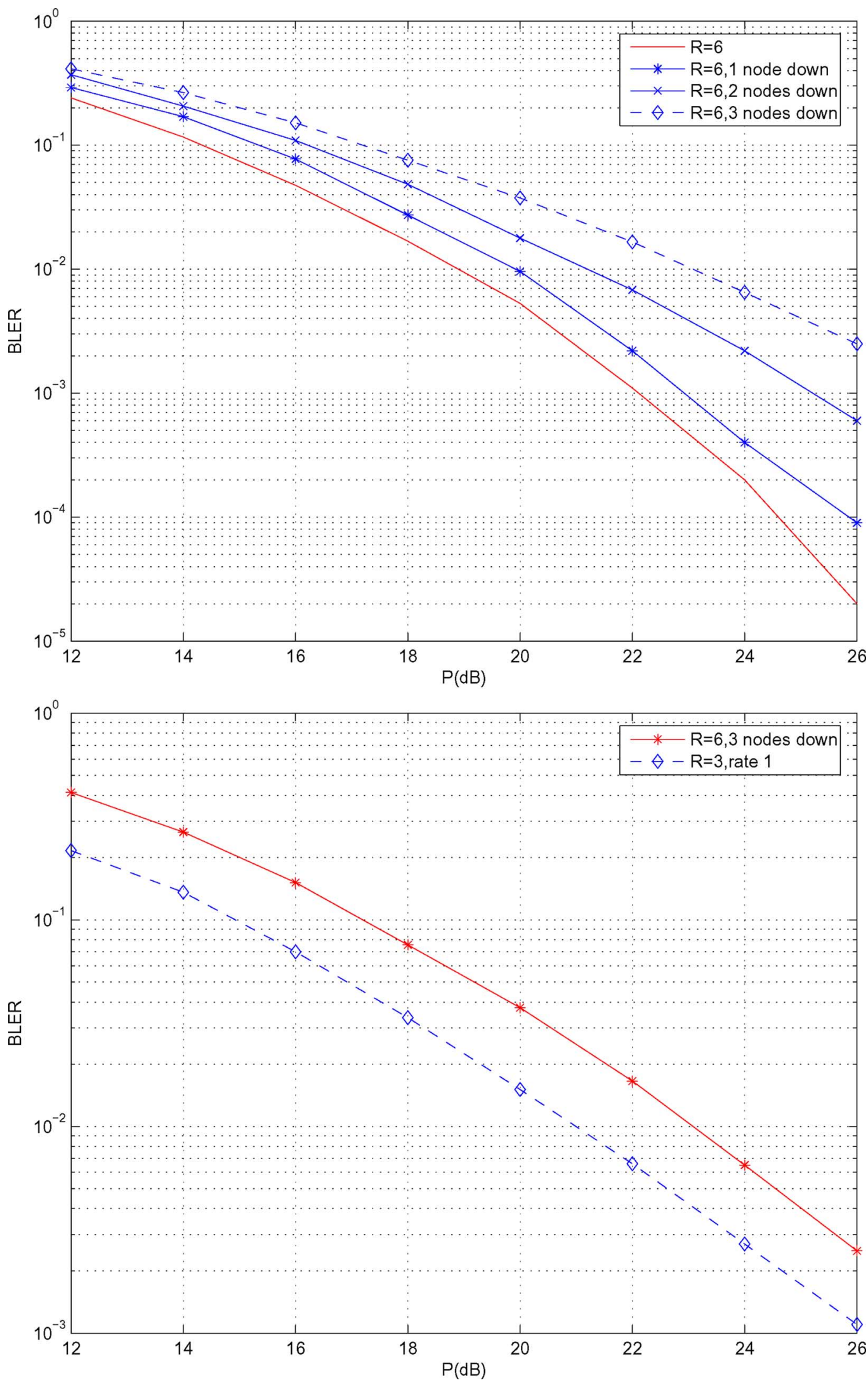

Fig. 6. On top, a network of six relay nodes with up to three nodes down, at the bottom, a six-nodes network with three nodes down versus a three-nodes network. 


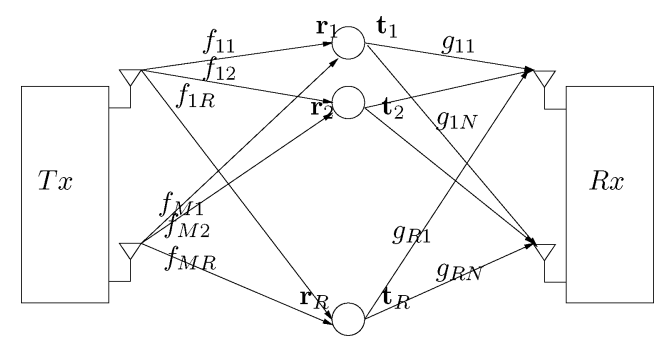

Fig. 7. A wireless network with multiple antenna nodes.

number of relays or the rate, one may need to have a faster algorithm. Recall that the mismatched decoder (11) is given by

$$
\begin{aligned}
\arg \min _{U_{l}, l=1, \ldots, L}\left\|\boldsymbol{y}(t+T)-U_{l} \boldsymbol{y}(t)\right\|^{2} \\
\quad=\arg \min _{l=1, \ldots, L} \sum_{k=1}^{R}\left|\boldsymbol{y}(t+T)_{k}-\zeta_{L}^{u_{k} l} \boldsymbol{y}(t)_{k}\right|^{2} .
\end{aligned}
$$

This minimization problem has already been studied in [3], where the authors show how the problem can be expressed as a lattice reduction problem. They give an algorithm to solve it, which is faster than exhaustive search.

\section{Generalization to Multiple-Antennas CASE}

In [11], a generalization of the network presented in Section II-A was given. In order to increase the data rate of the network, both the transmitter and receiver nodes are equipped with multiple antennas (see Fig. 7). Let $M$ and $N$ be the number of transmit, respectively, receive antennas. The codeword $\boldsymbol{S}$ sent at the transmitter is normalized such that $E\left[\operatorname{Tr}\left(\boldsymbol{S} \boldsymbol{S}^{\dagger}\right)\right]=M$. Channels are denoted by $f_{m j}$ from the $m$ th transmit antenna to the $j$ th relay and $g_{j n}$ from the $j$ th relay to the $n$th receive antenna (see Fig. 7), which are assumed to be independent and identically distributed (i.i.d.) $\mathcal{C N}(0,1)$. Similarly, let $\boldsymbol{v}_{i}$ and $W_{i}$ be the noise vectors, with coefficients also i.i.d. $\mathcal{C N}(0,1)$. Matrices at the relays are denoted by $A_{i}, i=1, \ldots, R$, and need to be unitary, as before. The power at the transmitter for each transmission is $P_{1}$, thus $P_{1} / M$ for each antenna. Each relay has a power of $P_{2} / R$ for each transmission.

By defining

$$
\begin{aligned}
Y & =\left[\boldsymbol{y}_{1}, \boldsymbol{y}_{2}, \ldots, \boldsymbol{y}_{N}\right] \\
S & =\left[A_{1} \boldsymbol{S} A_{2} \boldsymbol{S} \cdots A_{R} \boldsymbol{S}\right] \\
\boldsymbol{g}_{i} & =\left[g_{i 1}, g_{i 2}, \ldots, g_{i N}\right] \\
H & =\left[\boldsymbol{f}_{1} \boldsymbol{g}_{1}, \ldots, \boldsymbol{f}_{R} \boldsymbol{g}_{R}\right]^{T} \\
W & =\left[\sqrt{\frac{P_{2}}{P_{1}+1}} \sum_{i=1}^{R} g_{i j} A_{i} \boldsymbol{v}_{i}+\boldsymbol{w}_{j}\right]_{j=1}^{N}
\end{aligned}
$$

it was shown in [11] that the channel model can be written as

$$
Y=\sqrt{\frac{P_{1} P_{2} T}{\left(P_{1}+1\right) M}} S H+W .
$$

The received matrix $Y$ is a $T \times N$ matrix, while $S$ is a $T \times M R$ matrix, since the $A_{i}, i=1, \ldots, R$, are $T \times T$ matrices, and $\boldsymbol{S}$ is a $T \times M$ matrix.

We now show how the noncoherent scheme presented previously generalizes to the multiple-antenna scenario.

\section{A. The Noncoherent Channel}

Similarly to what we have done in Section II-B, we now need to design the matrices $A_{i}, i=1, \ldots, R$ and the codebook such that $S=\left[A_{1} \boldsymbol{S}, \ldots, A_{R} \boldsymbol{S}\right]$ is unitary, i.e., $S^{\dagger} S=\boldsymbol{I}_{M R}$.

First, we choose the codewords to be of the form

$$
\boldsymbol{S}_{j}=U_{j} \boldsymbol{S}^{\prime}, \quad j=1, \ldots, L
$$

where $U_{j}$ is a unitary $T \times T$ matrix, and $\boldsymbol{S}^{\prime}$ is an initial transmitted signal normalized to that $E\left[\operatorname{Tr}\left(\boldsymbol{S}_{j}^{\dagger} \boldsymbol{S}_{j}\right)\right]=M$ for all $j=1, \ldots, L$. As for the one-antenna case, we require that both the unitary matrices $A_{i}, i=1, \ldots, R$, and $U_{j}, j=1, \ldots, L$ be diagonal. Thus, denoting by $S_{j}=\left[A_{1} \boldsymbol{S}_{j}, \ldots, A_{R} \boldsymbol{S}_{j}\right]$, we have

$$
\begin{aligned}
S_{j}^{\dagger} S_{j} & =\left[A_{1} U_{j} \boldsymbol{S}^{\prime}, \ldots, A_{R} U_{j} \boldsymbol{S}^{\prime}\right]^{\dagger}\left[A_{1} U_{j} \boldsymbol{S}^{\prime}, \ldots, A_{R} U_{j} \boldsymbol{S}^{\prime}\right] \\
& =\left[A_{1} \boldsymbol{S}^{\prime}, \ldots, A_{R} \boldsymbol{S}^{\prime}\right]^{\dagger} U_{j}^{\dagger} U_{j}\left[A_{1} \boldsymbol{S}^{\prime}, \ldots, A_{R} \boldsymbol{S}^{\prime}\right] \\
& =\left[A_{1} \boldsymbol{S}^{\prime}, \ldots, A_{R} \boldsymbol{S}^{\prime}\right]^{\dagger}\left[A_{1} \boldsymbol{S}^{\prime}, \ldots, A_{R} \boldsymbol{S}^{\prime}\right]
\end{aligned}
$$

and the fact that $S_{j}$ is unitary only depends on the choice of the matrices at the relays and the transmitted signal $\boldsymbol{S}^{\prime}$. We thus design the matrices $A_{i}$ and $\boldsymbol{S}^{\prime}$ independently of the codebook of matrices $U_{j}$.

Suppose now that we have $T=M R$, with $M$ the number of transmit antennas, $R$ the number of relays, and $T$ the coherence time. Choose a first generalized GBH matrix $A$ of size $R \times R$, and a second one $B$ of size $M$ (cf. Definition 1), both with coefficients that are roots of unity. Take

$$
A_{i}=\operatorname{diag}(\underbrace{a_{1 i}, \ldots, a_{1 i}}_{M}, \ldots, \underbrace{a_{R i}, \ldots, a_{R i}}_{M})
$$

which is unitary for all $i$ since all $a_{i j}$ 's are roots of unity, and similarly

$$
\boldsymbol{S}^{\prime}=\frac{\sqrt{M}}{\left\|\boldsymbol{S}^{\prime}\right\|_{F}}\left(\begin{array}{ccc}
b_{1,1} & \ldots & b_{1, M} \\
\vdots & & \vdots \\
b_{M, 1} & & b_{M, M} \\
\vdots & & \vdots \\
b_{1,1} & \ldots & b_{1, M} \\
\vdots & & \vdots \\
b_{M, 1} & & b_{M, M}
\end{array}\right)
$$

where $\frac{\sqrt{M}}{\left\|S^{\prime}\right\|_{F}}$ is the normalization factor such that $E\left[\operatorname{Tr}\left(\boldsymbol{S}^{\prime \prime} \boldsymbol{S}^{\prime}\right)\right]=M$. By definition of the Frobenius norm $\|\cdot\|_{F}$

$$
\left\|\boldsymbol{S}^{\prime}\right\|_{F}=\sqrt{\sum_{i=1}^{M} \sum_{j=1}^{T}\left|s_{i, j}^{\prime}\right|^{2}}=\sqrt{M T}
$$

since all coefficients $s_{i, j}^{\prime}$ are roots of unity. By construction

$$
\left[A_{1} \boldsymbol{S}^{\prime}, \ldots, A_{R} \boldsymbol{S}^{\prime}\right]=\frac{1}{\sqrt{T}} A \otimes B
$$


and

$$
\left[A_{1} \boldsymbol{S}^{\prime}, \ldots, A_{R} \boldsymbol{S}^{\prime}\right]^{\dagger}\left[A_{1} \boldsymbol{S}^{\prime}, \ldots, A_{R} \boldsymbol{S}^{\prime}\right]=\frac{R M}{T} \boldsymbol{I}_{R M}=\boldsymbol{I}_{R M}
$$

since $A \otimes B$ is a GBH matrix of size $R M$.

Example 3: Let $M=2$ and $R=3$. Choose the following matrices:

$$
A=\left(\begin{array}{ccc}
1 & 1 & 1 \\
1 & \zeta_{3} & \zeta_{3}^{2} \\
1 & \zeta_{3}^{2} & \zeta_{3}
\end{array}\right) \text { and } B=\left(\begin{array}{cc}
1 & 1 \\
1 & -1
\end{array}\right)
$$

The unitary matrices at the relays are given by

$$
\begin{aligned}
& A_{1}=\boldsymbol{I}_{6} \\
& A_{2}=\operatorname{diag}\left(1,1, \zeta_{3}, \zeta_{3}, \zeta_{3}^{2}, \zeta_{3}^{2}\right) \\
& A_{3}=\operatorname{diag}\left(1,1, \zeta_{3}^{2}, \zeta_{3}^{2}, \zeta_{3}, \zeta_{3}\right)
\end{aligned}
$$

where $\boldsymbol{I}_{6}$ denote the identity matrix of size 6 , and the initial transmitted signal is

$$
\boldsymbol{S}^{\prime}=\sqrt{\frac{2}{12}}\left(\begin{array}{cc}
1 & 1 \\
1 & -1 \\
1 & 1 \\
1 & -1 \\
1 & 1 \\
1 & -1
\end{array}\right)
$$

\section{B. Decoding and Diversity}

A differential encoder is done similarly to the one-antenna case, so that a natural candidate for the differential decoder is

$$
\arg \min _{U_{l}, l=1, \ldots, L}\left\|Y(t+T)-U_{l} Y(t)\right\|^{2} .
$$

Let us restrict to the case where $T=M R$. Similarly to the single-antenna case, in order to analyze this strategy, we consider two instances of the noncoherent channel

$$
Y(t)=\sqrt{c_{\rho}} S(t) H+W(t)
$$

where $H$ is an $T \times N$ matrix unknown at both the transmitter and receiver, $S(t)$ is a $T \times T$ unitary matrix, and $c_{\rho}$ is a constant which depends on the $\operatorname{SNR} \rho$, that is

$$
\left(\begin{array}{c}
Y(t) \\
Y(t+T)
\end{array}\right)=\sqrt{c_{\rho}}\left(\begin{array}{c}
S(t) \\
S(t+T)
\end{array}\right) H+\left(\begin{array}{c}
W(t) \\
W(t+T)
\end{array}\right) .
$$

Since $S(t)$ and $S(t) \psi$ are indistinguishable for an arbitrary unitary $T \times T$ matrix $\psi$, we preprocess the signal so that

$$
\left(\begin{array}{c}
Y(t) \\
Y(t+T)
\end{array}\right)=\sqrt{c_{\rho}}\left(\begin{array}{c}
\boldsymbol{I}_{T} \\
U_{k}
\end{array}\right) H+\left(\begin{array}{c}
W(t) \\
W(t+T)
\end{array}\right)
$$

for $U_{k}$ a unitary matrix belonging to the codebook. To suit the network model, we have

$$
\begin{aligned}
W(t)=\left[\sqrt{\frac{P_{2}}{P_{1}+1}} \sum_{i=1}^{R} g_{i 1} A_{i} \boldsymbol{v}_{i}(t)+\boldsymbol{w}_{1}(t), \ldots,\right. \\
\\
\left.\sqrt{\frac{P_{2}}{P_{1}+1}} \sum_{i=1}^{R} g_{i N} A_{i} \boldsymbol{v}_{i}(t)+\boldsymbol{w}_{N}(t)\right]
\end{aligned}
$$

$H=\left[\boldsymbol{f}_{1} \boldsymbol{g}_{1}, \ldots, \boldsymbol{f}_{R} \boldsymbol{g}_{R}\right]^{T}$, with $\boldsymbol{f}_{i}=\left[f_{1 i}, \ldots, f_{M i}\right]^{T}$, and $\boldsymbol{g}_{i}=$ $\left[g_{i 1}, g_{i 2}, \ldots, g_{i N}\right]$. Furthermore, we have $c_{\rho}=\frac{P_{2} P_{1} T}{\left(P_{1}+1\right) M}$, and we denote $c_{\rho}^{\prime}=\frac{P_{2}}{P_{1}+1}$. Recall that $P_{1}=\rho / 2, P_{2}=\rho /(2 R)$, and $\rho$ is the total power of the system. Since the columns of the matrix $H$ are dependent, we cannot treat the channel equation in matrix form and have to vectorize it to do the analysis. We denote by $\operatorname{vec}(Y(t))$ the $T N \times 1$ vectorized version of $Y(t)$. Set $\boldsymbol{Y}=[\operatorname{vec}(Y(t)), \operatorname{vec}(Y(t+T))]^{t}$. The pairwise probability of error is thus given by

$$
\begin{aligned}
P( & \left.U_{k} \rightarrow U_{l}\right) \\
& =P\left(\left\|\operatorname{vec}(Y(t+T))-\left(U_{k} \otimes \boldsymbol{I}_{N}\right) \operatorname{vec}(Y(t))\right\|^{2}\right. \\
& \left.\geq\left\|\operatorname{vec}(Y(t+1))-\left(U_{l} \otimes \boldsymbol{I}_{N}\right) \operatorname{vec}(Y(t))\right\|^{2} \mid U_{k} \text { sent }\right) \\
& =P\left(\left\|\left[-\left(U_{k} \otimes \boldsymbol{I}_{N}\right) \boldsymbol{I}_{T N}\right] \boldsymbol{Y}\right\|^{2}\right. \\
& \left.\geq\left\|\left[-\left(U_{l} \otimes \boldsymbol{I}_{N}\right) \boldsymbol{I}_{T N}\right] \boldsymbol{Y}\right\|^{2} \mid U_{k} \text { is sent }\right) \\
& =P\left(\boldsymbol{Y}^{\dagger} \boldsymbol{\Delta} \boldsymbol{Y} \geq 0 \mid U_{k} \text { is sent }\right) \\
& =E_{\boldsymbol{g}} P\left(\boldsymbol{Y}^{\dagger} \boldsymbol{\Delta} \boldsymbol{Y} \geq 0 \mid \boldsymbol{g}\right)
\end{aligned}
$$

with $k \neq l$, and

$$
\boldsymbol{\Delta}=\left(\begin{array}{cc}
0 & \left(U_{l}^{\dagger}-U_{k}^{\dagger}\right) \otimes \boldsymbol{I}_{N} \\
\left(U_{l}-U_{k}\right) \otimes \boldsymbol{I}_{N} & 0
\end{array}\right) .
$$

We now use the same approach as in the single-antenna case and compute $P\left(\boldsymbol{Y}^{\dagger} \boldsymbol{\Delta} \boldsymbol{Y} \geq 0\right)$ knowing $\boldsymbol{g}$.

Proposition 2: We have that

$$
P\left(\boldsymbol{Y}^{\dagger} \Delta \boldsymbol{Y} \geq 0 \mid g\right)=\frac{1}{2 \pi i} \int_{-\infty}^{\infty} \frac{1}{\omega \operatorname{det}\left(\boldsymbol{I}_{2 T N}-i \omega \Delta \Sigma\right)} d \omega
$$

where $\Sigma$ is defined by (16).

Proof: We have

$$
\begin{aligned}
P\left(\boldsymbol{Y}^{\dagger} \boldsymbol{\Delta} \boldsymbol{Y} \geq 0\right) & =E\left[u\left(\boldsymbol{Y}^{\dagger} \boldsymbol{\Delta} \boldsymbol{Y}\right)\right] \\
& =E\left[\frac{1}{2 \pi} \int_{-\infty}^{\infty} \frac{e^{i \boldsymbol{Y}^{\dagger} \boldsymbol{\Delta}}}{i \omega} d \omega\right]
\end{aligned}
$$

where $u$ is the step function $(u(x)=1$ if $x>0, u(x)=$ 0 else), and the second equality is the Fourier transform of $u$. Computing the expectation yields

$$
\begin{aligned}
& P\left(\boldsymbol{Y}^{\dagger} \boldsymbol{\Delta} \boldsymbol{Y} \geq 0 \mid \boldsymbol{g}\right) \\
& =\frac{1}{2 \pi} \iint_{-\infty}^{\infty} \frac{e^{i \boldsymbol{Y}^{\dagger} \Delta \boldsymbol{Y}}}{i \omega} \frac{e^{-\boldsymbol{Y}^{\dagger} \Sigma^{-1} \boldsymbol{Y}}}{(2 \pi)^{2 T} \operatorname{det}(\Sigma)} d \omega d \boldsymbol{Y} \\
& =\frac{1}{(2 \pi)^{2 T N+1} i} \int_{-\infty}^{\infty} \int \frac{e^{-\boldsymbol{Y}^{\text {vec }}\left(-i \omega \Delta+\Sigma^{-1}\right) \boldsymbol{Y}^{\text {vec }}}}{\omega \operatorname{det}(\Sigma)} d \boldsymbol{Y} d \omega
\end{aligned}
$$

where $\Sigma=E\left[Y Y^{\dagger}\right]$ is the covariance matrix of $\boldsymbol{Y}$. Since the matrix $H$ can be written

$$
H=\left[\mathcal{G}_{1} f, \ldots, \mathcal{G}_{N} f\right]
$$


with $\boldsymbol{f}=\left[\boldsymbol{f}_{1}, \ldots, \boldsymbol{f}_{R}\right]^{t}$ and $\mathcal{G}_{n}=\operatorname{diag}\left(g_{1 n} \boldsymbol{I}_{M}, \ldots, g_{R n} \boldsymbol{I}_{M}\right)$, we have that

$\Sigma=\left(\begin{array}{rrr}\left(c_{\rho}^{\prime} \sum_{i=1}^{R}\left|g_{i 1}\right|^{2}+1\right) \boldsymbol{I}_{T} & & \\ & \ddots & \\ & & \left(c_{\rho}^{\prime} \sum_{i=1}^{R}\left|g_{i N}\right|^{2}+1\right) \boldsymbol{I}_{T}\end{array}\right)$

with

$$
\mathcal{G}=\left(\begin{array}{c}
\mathcal{G}_{1} \\
\vdots \\
\mathcal{G}_{N}
\end{array}\right)
$$

The imaginary part of $-\boldsymbol{Y}^{\dagger}\left(-i \omega \boldsymbol{\Delta}+\Sigma^{-1}\right) \boldsymbol{Y}$ is $i \boldsymbol{Y}^{\dagger} \omega \boldsymbol{\Delta} \boldsymbol{Y}$ since $\omega \Delta$ is Hermitian, while $\Sigma^{-1}$ is positive definite. Thus, the above integral converges, and we have the desired result.

We now derive the diversity in the asymptotic regime where we assume that both the power of the system $\rho$ and the number of relays $R$ are big. Recall that

$$
\begin{aligned}
& P\left(U_{k} \rightarrow U_{l}\right) \\
& =E_{\boldsymbol{g}} P\left(\boldsymbol{Y}^{\dagger} \boldsymbol{\Delta} \boldsymbol{Y} \geq 0 \mid \boldsymbol{g}\right) \\
& =E_{\boldsymbol{g} \mid \boldsymbol{g} \in \mathcal{A}} P\left(\boldsymbol{Y}^{\dagger} \boldsymbol{\Delta} \boldsymbol{Y} \geq 0\right)+E_{\boldsymbol{g} \mid \boldsymbol{g} \notin \mathcal{A}} P\left(\boldsymbol{Y}^{\dagger} \boldsymbol{\Delta} \boldsymbol{Y} \geq 0\right)
\end{aligned}
$$

where

$$
\mathcal{A}=\left\{\boldsymbol{g}||(1 / R) \sum_{i=1}^{R}\left|g_{i j}\right|^{2}-1 \mid<\epsilon \text { for all } j\right\} .
$$

When $R \rightarrow \infty$, we now show that $P(g \in \mathcal{A}) \rightarrow 1$ for all $\epsilon>0$, which will thus imply that

$$
E_{\boldsymbol{g} \mid \boldsymbol{g} \notin \mathcal{A}} P\left(\boldsymbol{Y}^{\dagger} \Delta \boldsymbol{Y} \geq 0\right) \rightarrow 0 .
$$

For any $j=1, \ldots, N$, we have

$$
\begin{aligned}
P\left(\left.\left|(1 / R) \sum_{i=1}^{R}\right| g_{i j}\right|^{2}-1 \mid\right. & <\epsilon) \\
& =1-P\left(\left.\left|(1 / R) \sum_{i=1}^{R}\right| g_{i j}\right|^{2}-1 \mid \geq \epsilon\right) .
\end{aligned}
$$

Since $\gamma:=\sum_{i=1}^{R}\left|g_{i j}\right|^{2}$ has a gamma distribution, we have that

$$
P(\gamma \geq R+\epsilon R)=\int_{R(1+\epsilon)}^{\infty} \frac{\gamma^{R-1} e^{-\gamma}}{(R-1) !} d \gamma
$$

which can be bounded, using a Chernoff bound, by

$$
\begin{aligned}
& P(\gamma \geq R+\epsilon R) \\
& \leq E\left[e^{\alpha(\gamma-R(1+\epsilon))}\right], \quad \alpha>0 \\
& =e^{-\alpha R(1+\epsilon)} \int_{0}^{\infty} \frac{\gamma^{R-1} e^{-\gamma(1-\alpha)}}{(R-1) !} d \gamma, \quad 0<\alpha<1 \\
& =e^{-\alpha R(1+\epsilon)} \int_{0}^{\infty}\left(\frac{u}{1-\alpha}\right)^{R-1} \frac{e^{-u}}{(R-1) !} \frac{d u}{1-\alpha}, \\
& u=(1-\alpha) \gamma \\
& =\left(\frac{e^{-\alpha(1+\epsilon)}}{1-\alpha}\right)^{R} .
\end{aligned}
$$

By computing the derivative of the above expression, we find that the optimal $\alpha$ is given by $1-\alpha=1 /(1+\epsilon)$, that is, $\alpha=\epsilon /(1+\epsilon)$, so that

$$
1-P(\gamma \geq R+\epsilon R) \geq 1-\left((1+\epsilon) e^{-\epsilon}\right)^{R} .
$$

Since $(1+\epsilon) e^{-\epsilon}<1$ for $\epsilon>0$, we have that $(1+\epsilon) e^{-\epsilon} \rightarrow 0$ with an exponential decay.

Proposition 3: We have that

$$
\begin{aligned}
& P\left(\boldsymbol{Y}^{\dagger} \Delta \boldsymbol{Y} \geq 0 \mid \boldsymbol{g} \in \mathcal{A}\right) \\
& \quad \leq \frac{1}{2} \operatorname{det}\left(\boldsymbol{I}_{T}+\frac{c_{\rho}}{16} \sum_{j=1}^{N} \mathcal{G}_{j} \mathcal{G}_{j}^{\dagger}\left(U_{k}-U_{l}\right)\left(U_{k}-U_{l}\right)^{\dagger}\right)^{-1} .
\end{aligned}
$$

Proof: We have that $c_{\rho}^{\prime} \sum_{i=1}^{R}\left|g_{i j}\right|^{2}+1 \approx 2$ since $c_{\rho}^{\prime}=$ $\rho /(R(\rho+2))$ and we are integrating over $\mathcal{A}$, which yields

$$
\Sigma \approx 2 \boldsymbol{I}_{2 T N}+c_{\rho}\left(\begin{array}{c}
\mathcal{G} \\
\mathcal{G} U_{k}
\end{array}\right)\left(\begin{array}{ll}
\mathcal{G}^{\dagger} & \mathcal{G}^{\dagger} U_{k}^{\dagger}
\end{array}\right)
$$

and what we need to compute is

$$
\operatorname{det}\left(\begin{array}{cc}
\boldsymbol{I}_{T N}-i \omega c_{\rho} \mathcal{G} \Delta^{\dagger} U_{k} \mathcal{G}^{\dagger} & -i \omega\left[2 \Delta^{\dagger}+c_{\rho} \mathcal{G} \Delta^{\dagger} \mathcal{G}^{\dagger}\right] \\
-i \omega\left[2 \Delta+c_{\rho} \mathcal{G} \Delta \mathcal{G}^{\dagger}\right] & \boldsymbol{I}_{T N}-i \omega c_{\rho} \mathcal{G} \Delta U_{k}^{\dagger} \mathcal{G}^{\dagger}
\end{array}\right)
$$

where $\Delta=U^{l}-U^{k}$. Since $\boldsymbol{I}_{T N}-i \omega c_{\rho} \mathcal{G} \Delta U_{k}^{\dagger} \mathcal{G}^{\dagger}$ and $-i \omega[2 \Delta+$ $\left.c_{\rho} \mathcal{G} \Delta \mathcal{G}^{\dagger}\right]$ commute, we have that this determinant is given by

$$
\operatorname{det}\left(\boldsymbol{I}_{T N}+c_{\rho} \mathcal{G}\left[i \omega \Delta \Delta^{\dagger}+\frac{4 \omega^{2} \Delta^{\dagger} \Delta}{c_{\rho}}+2 \omega^{2} 2 \Delta \Delta^{\dagger}\right] \mathcal{G}^{\dagger}\right) .
$$

Since $\rho \rightarrow \infty$, we have that $\frac{4 \omega^{2} \Delta^{\dagger} \Delta}{c_{\rho}} \rightarrow 0$, and the determinant simplifies to

$$
\begin{aligned}
& \operatorname{det}\left(\boldsymbol{I}_{T N}+c_{\rho} \mathcal{G} \Delta \Delta^{\dagger}\left[i \omega+4 \omega^{2}\right] \mathcal{G}^{\dagger}\right) \\
& =\operatorname{det}\left(\boldsymbol{I}_{T}+c_{\rho} \mathcal{G} \Delta \Delta^{\dagger}\left[i \omega+4 \omega^{2}\right] \sum_{j=1}^{N} \mathcal{G}_{j}^{\dagger} \mathcal{G}_{j}\right) \\
& =\prod_{k=1}^{T}\left(1+c_{\rho} \Delta_{k} \Delta_{k}^{\dagger}\left[i \omega+4 \omega^{2}\right] \sum_{j=1}^{N} \mathcal{G}_{j k}^{\dagger} \mathcal{G}_{j k}\right) \\
& =\prod_{k=1}^{T} 4 c_{\rho} \Delta_{k} \Delta_{k}^{\dagger} \sum_{j=1}^{N} \mathcal{G}_{j k}^{\dagger} \mathcal{G}_{j k}\left(\frac{1}{4 c_{\rho} \Delta_{k} \Delta_{k}^{\dagger} \sum_{j=1}^{N} \mathcal{G}_{j k}^{\dagger} \mathcal{G}_{j k}}\right. \\
& =\prod_{k=1}^{T} 4 c_{\rho} \Delta_{k} \Delta_{k}^{\dagger} \sum_{j=1}^{N} \mathcal{G}_{j k}^{\dagger} \mathcal{G}_{j k}\left(\left(\omega+\frac{i \omega}{8}\right)^{2}+\omega^{2}\right)
\end{aligned}
$$

where

$$
c_{k}=\sqrt{\frac{1}{64}+\frac{1}{4 c_{\rho} \Delta_{k} \Delta_{k}^{\dagger} \sum_{j=1}^{N} \mathcal{G}_{j k}^{\dagger} \mathcal{G}_{j}}} .
$$

From now on, we can follow step by step the computation done for the single-antenna case, which finally yields that

$$
P\left(\boldsymbol{Y}^{\dagger} \Delta \boldsymbol{Y} \geq 0 \mid \boldsymbol{g}\right) \leq \frac{1}{2}\left(\prod_{k=1}^{T} c_{\rho} \Delta_{k} \Delta_{k}^{\dagger} \sum_{j=1}^{N} \mathcal{G}_{j k}^{\dagger} \mathcal{G}_{j} \frac{1}{16}+1\right)^{-1}
$$

which concludes the proof. 


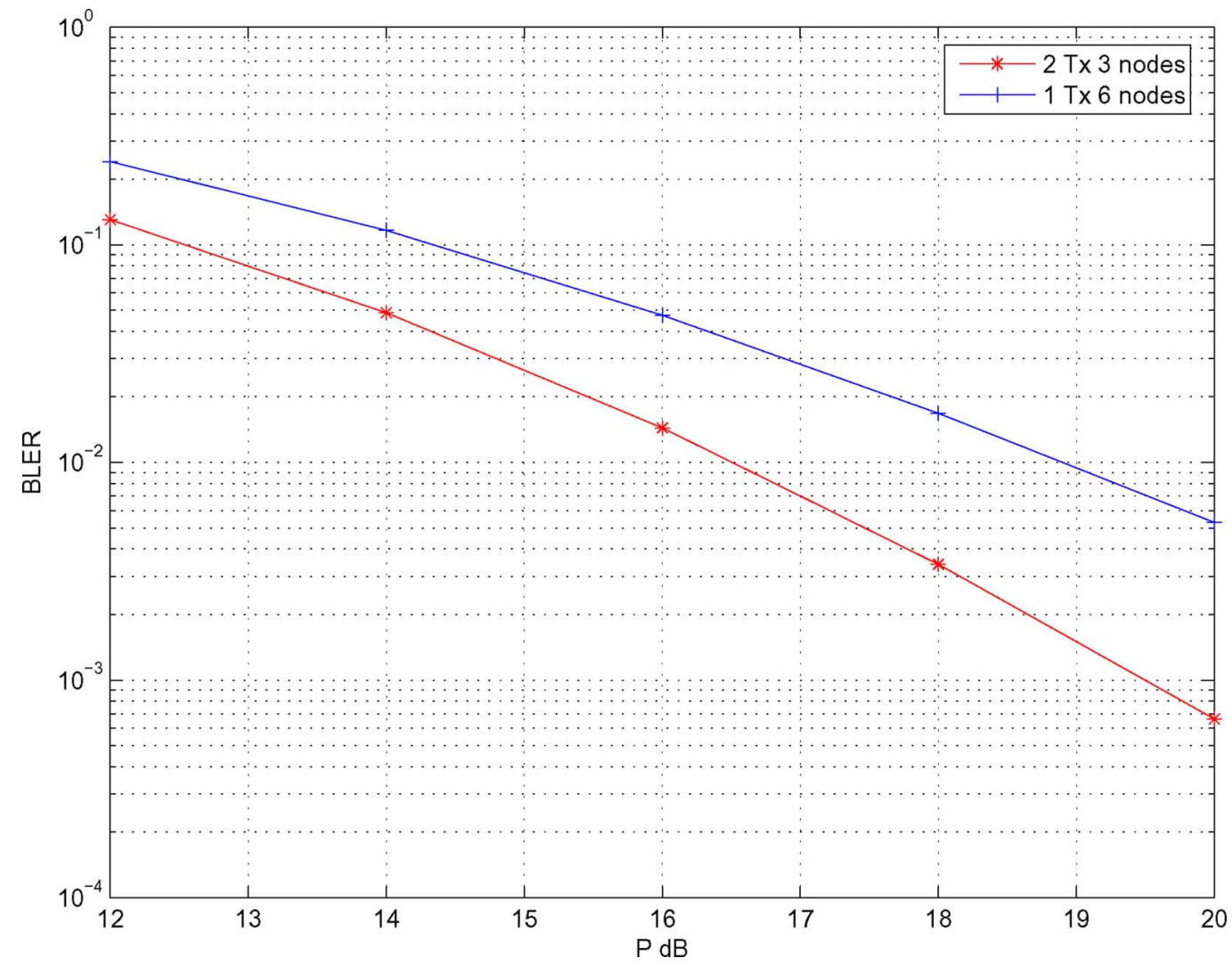

Fig. 8. A six- nodes network with one antenna versus a three-nodes network with two antennas.

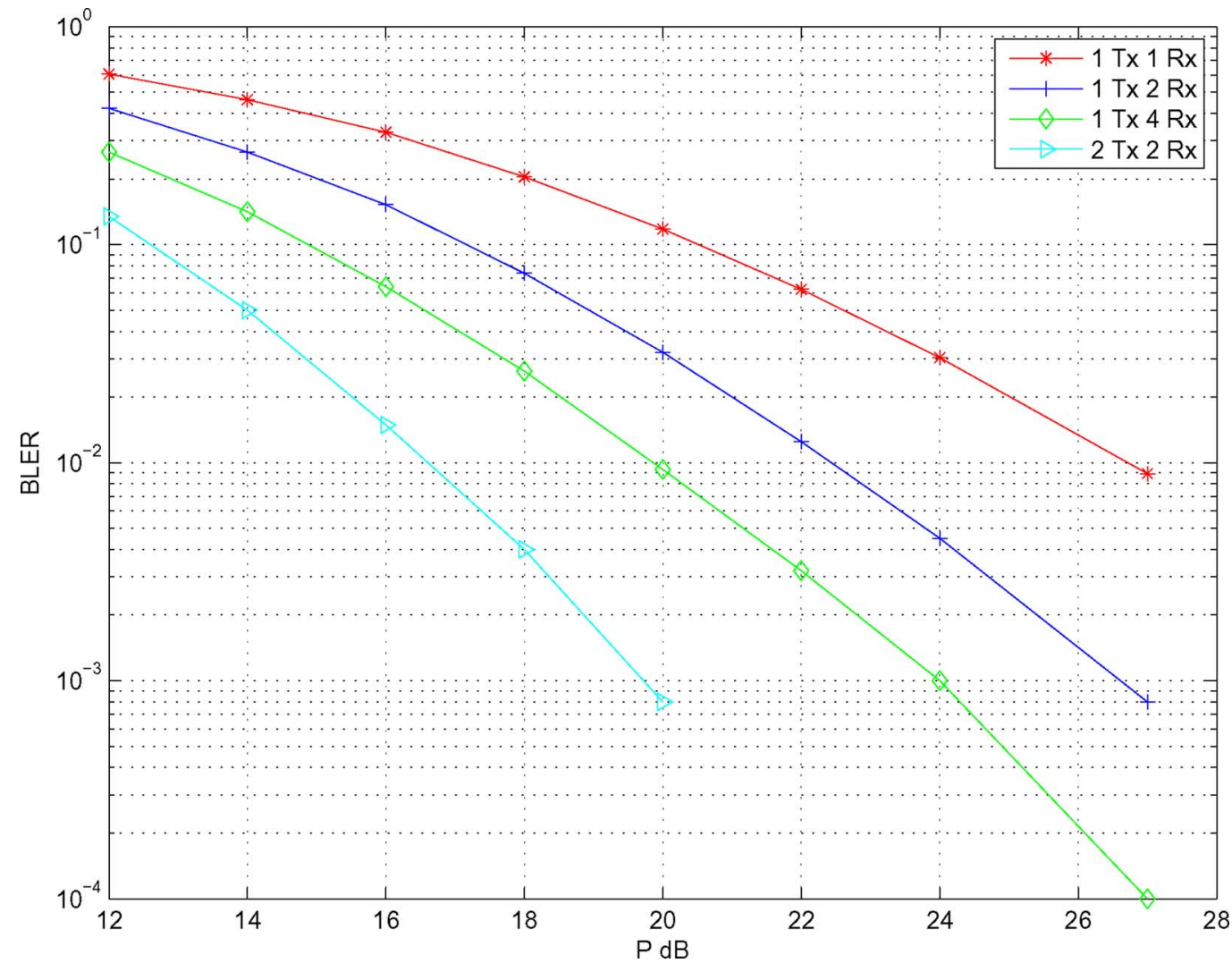

Fig. 9. A three-nodes network with $1 \mathrm{Tx}$ antenna and 1, 2, and $4 \mathrm{Rx}$ antennas. 
Thus

$$
\begin{aligned}
P\left(U_{k}\right. & \left.\rightarrow U_{l}\right)=P\left(\boldsymbol{Y}^{\dagger} \Delta \boldsymbol{Y} \geq 0\right) \\
& \leq E_{\boldsymbol{g} \mid \boldsymbol{g} \in \mathcal{A}} \operatorname{det}\left(\boldsymbol{I}_{T}+\frac{\rho}{32} \sum_{j=1}^{N} \mathcal{G}_{j} \mathcal{G}_{j}^{\dagger}\left(U_{k}-U_{l}\right)\left(U_{k}-U_{l}\right)^{\dagger}\right)^{-1}
\end{aligned}
$$

We now use the computation of the above expectation done in [11] to conclude that the diversity gain is given by

$$
d= \begin{cases}\min \{M, N\} R, & M \neq N \\ M R\left(1-\frac{1}{M} \frac{\log \log P}{\log P}\right), & M=N\end{cases}
$$

assuming again that $\left(U_{k}-U_{l}\right)\left(U_{k}-U_{l}\right)^{\dagger}$ is full rank (that is, the code is fully diverse).

Fig. 8 shows simulation results comparing the performance of a six-nodes network with one antenna at transmitter and receiver to a three-nodes network with two antennas at both transmitter and receiver nodes. The $x$ axis shows the power of the system in decibels, and the $y$ axis the BLER. We are interested in the diversity of both systems. Note that both networks use the same codebook. The diversity of the two-antennas network is however slightly better, which is expected looking at the expression of the diversity for both cases, since we have $R\left(1-\frac{\log \log P}{\log P}\right)$ versus $2 R\left(1-\frac{1}{2} \frac{\log \log P}{\log P}\right)$.

Fig. 9, a network with three nodes is considered, where the receiver is using $M=1$ antenna, while the receiver is using $N=$ 1,2 and 4 antennas. We see a clear coding gain by increasing the number of receive antennas. The second curve shows the performance with $1 \mathrm{Tx}$ and $2 \mathrm{Rx}$ antennas. One can compare the improvement of performance obtained in either choosing to add two more receive antenna (the third curve), or one more transmit antenna (the fourth curve). While there is indeed a gain in adding two receive antennas, it is nothing comparable with adding one transmit antenna (the theory predicts the same diversity order between the curves 2 and 3 , given by $\min (M, N) R=$ $\min (1,2) 3=\min (1,4) 3=3$, while the diversity order for the fourth curve is $\min (2,3) 3=6$ ).

\section{CONCLUSION}

We considered the problem of coding over a wireless relay network. While existing schemes heavily rely on the knowledge of the channel, either at both relays and receiver, or at least at the receiver, we presented a scheme that requires no channel knowledge. Furthermore, it is available for any number of relay nodes. This scheme is based on two ideas: distributed space-time coding, where relay nodes cooperate to encode the data, and differential MIMO coding, a popular technique to code over a noncoherent MIMO channel. We analyzed this strategy and showed that the diversity of the system depends on the number of relay nodes. Actually, when confronted to $d$-node failures, an $R$-nodes network behaves, in terms of diversity, as a network with $R-d$. Finally, we extended our construction to the case where both the transmitter and receiver nodes have several antennas.

\section{ACKNOWLEDGMENT}

The authors would like to thank M. Varanasi and Y. Jing for sending their preprints.

\section{REFERENCES}

[1] K. Azarian, H. El Gamal, and P. Schniter, "On the achievable diversity-multiplexing tradeoff in half-duplex cooperative channels," IEEE Trans. Inf. Theory, vol. 51, no. 12, pp. 4152-4172, Dec. 2005.

[2] H. Bölcskei, R. U. Nabar, Ö. Oyman, and A. J. Paulraj, "Capacity scaling laws in MIMO relay networks," IEEE Trans. Wireless Commun., vol. 5, no. 6, pp. 1433-1444, Jun. 2006.

[3] K. L. Clarkson, W. Sweldens, and A. Zheng, "Fast multiple antenna differential decoding," IEEE Trans. Commun., vol. 49, no. 2, pp. 253-261, Feb. 2001.

[4] P. Dayal and M. K. Varanasi, "Distributed QAM-based space-time block codes for efficient cooperative multiple-access communication," IEEE Trans. Inf. Theory, vol. 54, no. 9, pp. 4342-4354, Sep. 2008.

[5] P. Elia, K. Vinodh, M. Anand, and P. V. Kumar, "D-MG Tradeoff and optimal codes for a class of $\mathrm{AF}$ and $\mathrm{DF}$ cooperative communication protocols," IEEE Trans. Inf. Theory, vol. 55, no. 7, pp. 3161-3185, Jul. 2009.

[6] B. M. Hochwald and T. L. Marzetta, "Unitary space-time modulation for multiple-antenna communications in Rayleigh flat fading," IEEE Trans. Inf. Theory, vol. 46, no. 2, pp. 543-564, Mar. 2000.

[7] B. Hochwald and W. Sweldens, "Differential unitary space time modulation," IEEE Trans. Commun., vol. 48, no. 12, pp. 2041-2052, Dec. 2000.

[8] K. J. Horadam, "A generalised Hadamard transform," in Proc. IEEE Int. Symp. Information Theory (ISIT 05), Adelaide, Australia, Sep. 2005, pp. 1006-1008.

[9] B. Hughes, "Differential space-time modulation," IEEE Trans. Inf. Theory, vol. 46, no. 7, pp. 2567-2578, Nov. 2000.

[10] Y. Jing and B. Hassibi, "Distributed space-time coding in wireless relay networks," IEEE Trans. Wireless Commun., vol. 5, no. 12, pp. 3524-3536, Dec. 2006.

[11] Y. Jing and B. Hassibi, "Diversity analysis of distributed space-time codes in relay networks with multiple transmit/receive antennas," EURASIP J. Adv. Signal Process., vol. 2008, 2008, Article ID 254573; doi: $10.1155 / 2008 / 254573$.

[12] Y. Jing and H. Jafarkhani, "Using orthogonal and quasi-orthogonal designs in wireless relay networks," in Proc IEEE 2006 Globecom, San Francisco, CA, Nov./Dec. 2006

[13] Y. Jing and H. Jafarkhani, "Distributed differential space-time coding for wireless relay networks," IEEE Trans. Commun., vol. 56, no. 7, pp. 1092-1100, Jul. 2008

[14] K. T and B. S. Rajan, "Distributed space-time codes with reduced decoding complexity," in Proc. IEEE Int. Symp. Information Theory (ISIT), Seattle, WA, Jul. 2006, pp. 542-546.

[15] K. T and B. S. Rajan, "Partially-coherent distributed space-time codes with differential encoder and decoder," in Proc. IEEE Int. Symp. Information Theory (ISIT), Seattle, WA, Jul. 2006, pp. 547-551.

[16] G. Susinder Rajan and B. Sundar Rajan, "A non-orthogonal distributed space-time coded protocol Part I: Signal model and design criteria," in Proc. IEEE Information Theory Workshop (ITW), Chengdu, China, Oct. 2006, pp. 385-389.

[17] J. N. Laneman and G. W. Wornell, "Distributed space-time coded protocols for exploiting cooperative diversity in wireless networks," IEEE Trans. Inf. Theory, vol. 49, no. 10, pp. 2415-2525, Oct. 2003.

[18] F. Oggier and B. Hassibi, "An algebraic family of distributed spacetime codes for wireless relay networks," in Proc. IEEE Int. Symp. Information Theory (ISIT), Seattle, WA, Jul. 2006, pp. 538-541.

[19] F. Oggier and B. Hassibi, "An algebraic coding scheme for wireless relay networks with multiple-antenna nodes," IEEE Trans. Signal Process., vol. 56, no. 7, pt. I, pp. 2957-2966, Jul. 2008.

[20] F. Oggier and B. Hassibi, "A coding strategy for wireless networks with no channel information," in Proc. 44th Allerton Conf. Communication, Control and Computing, Monticello, IL, Sep. 2006.

[21] F. Oggier and B. Hassibi, "A coding scheme for wireless networks with multiple antenna nodes and no channel information," in Proc. IEEE Int. Conf, Acoustics, Speech and Signal Processing (ICASSP), Honolulu, HI, Apr. 2007.

[22] F. Oggier, "Design of algebraic cyclic codes," in Proc. IEEE Iformation Theory Workshop (ITW), Porto, Portugal, May 2008. 
[23] A. Shokrollahi, B. Hassibi, B. M. Hochwald, and W. Sweldens, "Representation theory for high-rate multiple-antenna code design," IEEE Trans. Inf. Theory, vol. 47, no. 6, pp. 2335-2367, Sep. 2001.

[24] S. Yang and J.-C. Belfiore, "Optimal space-time codes for the amplifyand-forward cooperative channel," IEEE Trans. Inf. Theory, vol. 53, no. 2, pp. 647-663, Feb. 2007.

[25] S. Yiu, R. Schober, and L. Lampe, "Differential distributed space-time block coding," in Proc. IEEE Pacific Rim Conf. Communications, Computers and Signal Processing, Victoria, BC, Canada, Aug. 2005.

Frédérique Oggier received the Diplôme degree in mathematics and computer science in 2000 from the University of Geneva, Switzerland. She then joined the Swiss Federal Institute of Technology, Lausanne (EPFL), where she graduated from the Doctoral School in Communication Systems (2001), and completed the $\mathrm{Ph} . \mathrm{D}$. dissertation in mathematics (2005).

She was a postdoctoral visitor at the California Institute of Technology $(\mathrm{Cal}-$ Tech), Pasadena, from 2005 to 2007, and at the Research Center for Information Security (RCIS), Tokyo, Japan, from 2007 to 2008. She is currently an Assistant Professor in the School of Physical and Mathematical Sciences, Nanyang Technological University (NTU), Singapore. Her main research interests are in applied algebra to coding problems arising in wireless communications, as well as information-theoretic security.

Prof. Oggier is a recipient of the Singapore NRF Fellowship.
Babak Hassibi was born in Tehran, Iran, in 1967. He received the B.S. degree from the University of Tehran in 1989 and the M.S. and Ph.D. degrees from Stanford University, Stanford, CA, in 1993 and 1996, respectively, all in electrical engineering.

He has been with the California Institute of Technology, Pasadena, since January 2001, where he is currently Professor and Executive Officer of Electrical Engineering. From October 1996 to October 1998, he was a Research Associate at the Information Systems Laboratory, Stanford University, and from November 1998 to December 2000, he was a Member of the Technical Staff in the Mathematical Sciences Research Center at Bell Laboratories, Murray Hill, NJ. He has also held short-term appointments at Ricoh California Research Center, the Indian Institute of Science, and Linköping University, Sweden. His research interests include wireless communications and networks, robust estimation and control, adaptive signal processing and linear algebra. $\mathrm{He}$ is the coauthor of the books (both with A. H. Sayed and T. Kailath) Indefinite Quadratic Estimation and Control: A Unified Approach to $H^{2}$ and $H^{\infty}$ Theories (New York: SIAM, 1999) and Linear Estimation (Englewood Cliffs, NJ: Prentice Hall, 2000).

Prof. Hassibi is a recipient of an Alborz Foundation Fellowship, the 1999 O. Hugo Schuck best paper award of the American Automatic Control Council (with H. Hindi and S. P. Boyd), the 2002 National Science Foundation Career Award, the 2002 Okawa Foundation Research Grant for Information and Telecommunications, the 2003 David and Lucille Packard Fellowship for Science and Engineering, and the 2003 Presidential Early Career Award for Scientists and Engineers (PECASE), and was a participant in the 2004 National Academy of Engineering "Frontiers in Engineering" program. He has been a Guest Editor for the IEEE TRANSACTIONS ON THEORY special issue on "Space-Time Transmission, Reception, Coding and Signal Processing" was an Associate Editor for Communications of the IEEE TRANSACTIONS ON INFORMATION THEORY during 2004-2006, and is currently an Editor for the Journal Foundations and Trends in Information and Communication. 